\title{
Viscous and inviscid models in fluid-particle interaction
}

\author{
Joshua Ballew* and Konstantina Trivisa ${ }^{\dagger}$
}

\begin{abstract}
Some recent developments in the study of fluid-particle interaction models are reviewed. A viscous model governed by the NavierStokes-Smoluchowski system and an inviscid model governed by the Euler-Smoluchowski system are presented and analyzed. For the viscous model, results on global existence, asymptotic behavior, singular limits and weak-strong uniqueness are discussed, whereas the analysis on the inviscid model deals with the issue of existence of smooth solutions.
\end{abstract}

2010 Mathematics SubJect Classifications: Primary 35Q84; secondary 35D30.

1 Introduction $\quad 46$

2 Viscous model: global existence results 51

2.1 Notions of weak solutions $\quad 51$

$\begin{array}{lll}2.2 & \text { Weak-strong uniqueness result } & 57\end{array}$

3 Viscous model: asymptotic analysis $\quad 58$

3.1 Low stratification low mach number limit $\quad 60$

3.2 Strong stratification and low mach number limit $\quad 66$

4 Inviscid models: main results $\quad 67$

$\begin{array}{lll}\text { 4.1 Reformulation of the problem } & 67\end{array}$

$\begin{array}{lll}4.2 & \text { Iterative method and local existence } & 68\end{array}$

$5 \quad$ Finite propagation speed $\quad 72$

*The work of J.B. was supported by the National Science Foundation under the grant DMS-1211638 and the Ann G. Wylie Dissertation Fellowship.

${ }^{\dagger}$ The work of K.T. was supported by the National Science Foundation under the Grant DMS-1211638 and by the Simmons Fellows in Mathematics Program \# 267399 . 


\section{Introduction}

Fluid-particle interaction is of great significance in sedimentation analysis of disperse suspensions of particles in fluids, one of the issues being the separation of the solid grains from the fluid by external forces: gravity settling processes or centrifugal forces. These procedures occur in many practical applications in biotechnology, medicine, waste-water recycling and mineral processing [9], as well as atmospheric science on issues related to pollution [41], and combustion dealing with reactive gases formed by fuel droplets [1, 2, 42, 43]. Aerosols and sprays can be modeled by fluid-particle-type interactions in which bubbles of suspended substances are seen as solid particles.

The coupling between the kinetic and the fluid equations is obtained through the friction forces that the fluid and the particles exert mutually. The friction force is assumed to follow Stokes law and thus is proportional to the relative velocity vector, i.e., is proportional to the fluctuations of the microscopic velocity $\xi \in \mathbb{R}^{3}$ around the fluid velocity field $\mathbf{u}$. More precisely, the cloud of particles is described by its distribution function $f_{\epsilon}(t, x, v)$ on phase space, which is the solution to the dimensionless Vlasov-Fokker-Planck equation

$(\mathrm{VFP}) \partial_{t} f_{\epsilon}+\frac{1}{\sqrt{\epsilon}}\left(v \cdot \nabla_{x} f_{\epsilon}-\nabla_{x} \Phi \cdot \nabla_{v} f_{\epsilon}\right)=\frac{1}{\epsilon} \operatorname{div}_{v}\left(\left(v-\sqrt{\epsilon} u_{\epsilon}\right) f+\nabla_{v} f_{\epsilon}\right)$.

Here, $\epsilon>0$ is a dimensionless parameter, whereas the drag force is independent of the fluid density $\varrho_{\epsilon}$, but proportional to the relative velocity of the fluid and the particles given by $v-u_{\epsilon}(t, x)$. The right hand-side of the moment equation in the Navier-Stokes system takes into account the action of the cloud of particles on the fluid through the forcing term

$$
F_{\epsilon}=\int_{\mathbb{R}^{3}}\left(\frac{v}{\sqrt{\epsilon}}-u_{\epsilon}(t, x)\right) f(t, x, v) d v .
$$

The density of the particles $\eta_{\epsilon}(t, x)$ is related to the probability distribution function $f_{\epsilon}(t, x, v)$ through the relation

$$
\eta_{\epsilon}(t, x)=\int_{\mathbb{R}^{3}} f_{\epsilon}(t, x, v) d v .
$$


There are two different scaling limits for this model, the so-called: bubbling and flowing regimes. They correspond to the diffusive approximation of the kinetic equation, bubbling regime, written in (VFP) and the strong drag force and strong Brownian motion for the flowing regime.

In this work we consider two prototype models for fluid particle interaction.

- A viscous model governed by the Navier-Stokes system for the evolution of a viscous, compressible fluid coupled with the so-called Smoluchowski equation used to monitor the evolution of particles within the fluid.

- An inviscid model governed by the Euler system for the evolution of an inviscid, compressible fluid coupled with the Smoluchowski equation for the evolution of particles dispersed in the fluid.

The governing equations for the viscous model express the conservation of mass, the balance of momentum, and the balance of particle densities often referred as the Smoluchowski equation:

$\partial_{t}(\varrho \mathbf{u})+\operatorname{div}_{x}(\varrho \mathbf{u} \otimes \mathbf{u})+\nabla_{x}\left(p_{F}(\varrho)+\eta\right)-\mu \Delta_{x} \mathbf{u}-\lambda \nabla_{x} \operatorname{div}_{x} \mathbf{u}=-(\eta+\beta \varrho) \nabla_{x} \Phi$

$$
\partial_{t} \eta+\operatorname{div}_{x}\left(\eta\left(\mathbf{u}-\nabla_{x} \Phi\right)\right)-\Delta_{x} \eta=0 .
$$

In the present context, the macroscopic variables are given by the density $\varrho$, the velocity field $\mathbf{u}$ and the particle density $\eta$. Here, $p_{F}$ denotes the pressure $p_{F}(\varrho)=a \varrho^{\gamma}, a>0, \gamma>1, \beta \neq 0$, and $\Phi$ denotes the external potential (typically incorporating gravity and boyancy).

In this work, we consider potentials that satisfy a suitable confinement condition (HC) (see Section 2). The total pressure $P=P(\varrho, \eta)$ in the mixture depends on the density of the particles and the density of the fluid and is given as a superposition of two parts

$$
P(\varrho, \eta)=p_{F}(\varrho)+\eta \text {. }
$$

The spatial domain $\Omega \subset \mathbb{R}^{3}$ is $C^{2, \nu}$ for some $\nu>0$ and can be bounded or unbounded. The viscosity parameters $\mu>0$ and $\lambda+\frac{2}{3} \mu \geq 0$ are nonnegative constants, while $\beta>0$ if $\Omega$ is unbounded.

We impose the no-slip boundary condition for the velocity vector leading to no-flux for the fluid density through the boundaries and the no-flux 
condition for the particle density

$$
\left.\mathbf{u}\right|_{\partial \Omega}=\nabla_{x} \eta \cdot \mathbf{n}+\eta \nabla_{x} \Phi \cdot \mathbf{n}=0 \text { on }(0, T) \times \partial \Omega,
$$

with $\mathbf{n}$ denoting the outer normal vector to the boundary $\partial \Omega$.

Our problem is supplemented with the initial data $\left\{\varrho_{0}, \mathbf{m}_{0}, \eta_{0}\right\}$ such that

$$
\left\{\begin{array}{l}
\varrho(0, x)=\varrho_{0} \in L^{\gamma}(\Omega) \cap L_{+}^{1}(\Omega) \\
(\varrho \mathbf{u})(0, x)=\mathbf{m}_{0} \in L^{\frac{6}{5}}(\Omega) \cap L^{1}(\Omega) \\
\eta(0, x)=\eta_{0} \in L^{2}(\Omega) \cap L_{+}^{1}(\Omega)
\end{array}\right.
$$

System (1.1)-(1.3) is obtained as $\epsilon \rightarrow 0$, by the standard Hilbert-expansion procedure, through the scaling limit in

$$
\left\{\begin{array}{l}
\partial_{t} f_{\epsilon}+\frac{1}{\sqrt{\epsilon}}\left(v \cdot \nabla_{x} f_{\epsilon}-\nabla_{x} \Phi \cdot \nabla_{v} f_{\epsilon}\right)=\frac{1}{\epsilon} \operatorname{div}_{v}\left(\left(v-\sqrt{\epsilon} u_{\epsilon}\right) f+\nabla_{v} f_{\epsilon}\right) \\
\partial_{t} \varrho_{\epsilon}+\operatorname{div}_{x}\left(\varrho_{\epsilon} u_{\epsilon}\right)=0 \\
\partial_{t}\left(\varrho_{\epsilon} u_{\epsilon}\right)+\operatorname{div}_{x}\left(\varrho_{\epsilon} u_{\epsilon} \otimes u_{\epsilon}\right)+\nabla_{x} p_{F}\left(\varrho_{\epsilon}\right)-\mu \Delta u+\beta \varrho_{\epsilon} \nabla_{x} \Phi=F_{\epsilon}
\end{array}\right.
$$

In this regime the particles are supposed to have negligible density with respect to the fluid, and thus, due to buoyancy effects, will typically move upwards in a system under gravity. From this phenomenon the regime bears the name of bubbling.

Two notions of weak solutions to system (1.1)-(1.3) are presented in the sequel (cf. Section 2); the concept of free energy solutions and the notion of weakly dissipative solutions. Analytical results on the global existence of free energy solutions to (1.1)-(1.3) have been established by Carrillo, Karper and Trivisa in [15]. This work presents a new approximating scheme for the construction of global solutions based on time-discretization.

Results on the global existence of weakly dissipative solutions to (1.1)(1.3) have been established by Ballew and Trivisa in [6]. The notion of weakly dissipative solutions in the context of compressible fluids relies on the construction of an approximate scheme based on an approximate relative entropy inequality. The introduction of this alternative notion of solutions arises from the desire to establish global solutions with greater regularity as well as weak-strong uniqueness results for models of compressible fluids exhibiting fluid-particle interaction.

The main ingredients of our approach can be summarized as follows:

(a) The introduction of an intrinsic definition of weakly dissipative solutions (known also as suitable weak solutions) to the Navier-Stokes- 
Smolukowski system, satisfying a relative entropy-type inequality. The form of this inequality is closely related to the energy of system.

(b) The construction of an explicit approximation scheme and the establishment of the existence of global in time suitable weak solutions for any finite energy initial data.

(c) The investigation of properties of suitable weak solutions, such as the problem of weak-strong uniqueness and conditional regularity issues.

A review of these results are presented in Section 2.

The analysis covers both bounded and unbounded domains in the threedimensional space $\mathbb{R}^{3}$. In the latter case, some delicate estimates are required for the establishment of the appropriate compactness of the approximate solutions. In that context, a confinement hypothesis on the domain $\Omega$ and potential $\Phi$ plays a crucial role in providing control of the negative contribution of the physical entropy in the free energy bounds for unbounded domains.

In addition, we show (cf. Carrillo, Karper and Trivisa [15]) that the total masses are conserved also in the large-time limit, which allows the unique determination of the long time asymptotics. A review of asymptotic analysis of weak solutions as well as results on singular limits are presented in Section 3.

The governing equations for the inviscid model express the conservation of mass, balance of momentum and balance of particle density

$$
\begin{gathered}
\partial_{t} \varrho+\operatorname{div}_{x}(\varrho \mathbf{u})=0, \\
\partial_{t}(\varrho \mathbf{u})+\operatorname{div}_{x}(\varrho \mathbf{u} \otimes \mathbf{u})+\nabla_{x}\left(p_{F}(\varrho)+\eta\right)=-(\eta+\beta \varrho) \nabla_{x} \Phi, \\
\partial_{t} \eta+\operatorname{div}_{x}\left(\eta\left(\mathbf{u}-\nabla_{x} \Phi\right)\right)-\Delta \eta=0 .
\end{gathered}
$$

The total pressure $P=P(\varrho, \eta)$ in the mixture depends on the density of the particles and the density of the fluid and is given by

$$
P(\varrho, \eta)=p_{F}(\varrho)+\eta
$$

with

$$
p_{F}(\varrho):=a \varrho^{\gamma}, \text { with } a>0 \text { and } \gamma>1 .
$$

The external potential

$$
\Phi: \Omega \rightarrow \mathbb{R}^{+}
$$

represents the effects of gravity and buoyancy and $\beta$ in (1.2) is taken to be positive. $\Omega$ is the spatial domain under consideration, which in the present 
context is the entire space $\mathbb{R}^{3}$. In this work we investigate the Cauchy problem for (1.1)-(1.3) with the initial conditions

$$
\left.(\varrho, \mathbf{u}, \eta)\right|_{t=o}=\left(\varrho_{0}, \mathbf{u}_{0}, \eta_{0}\right) .
$$

The Euler-Smoluchowski system (1.7)-(1.9) is derived formally as $\varepsilon \rightarrow 0$ in [13] by the standard Hilbert expansion procedure, through the scaling limit in

$$
\left\{\begin{array}{l}
\partial f_{\varepsilon}+\frac{1}{\sqrt{\varepsilon}}\left(\xi \cdot \nabla_{x} f_{\varepsilon}-\nabla_{x} \Phi \cdot \nabla_{\xi} f_{\varepsilon}\right)=\frac{1}{\varepsilon} \operatorname{div}_{\xi}\left(\left(\xi-\sqrt{\varepsilon} \mathbf{u}_{\varepsilon}\right) f+\nabla_{\xi} f_{\varepsilon}\right), \\
\partial_{t} \varrho_{\varepsilon}+\operatorname{div}_{x}\left(\varrho_{\varepsilon} \mathbf{u}\right)=0, \\
\partial_{t}\left(\varrho_{\varepsilon} \mathbf{u}_{\varepsilon}\right)+\operatorname{div}_{x}\left(\varrho_{\varepsilon} \otimes \mathbf{u}_{\varepsilon}\right)+\chi \nabla_{x} p_{F}\left(\varrho_{\varepsilon}\right)+\operatorname{sign}(\alpha) \varrho_{\varepsilon} \nabla_{x} \Phi=\left(J_{\varepsilon}-\eta_{\varepsilon} \mathbf{u}_{\varepsilon}\right) .
\end{array}\right.
$$

with

$$
\eta_{\varepsilon}=\int_{\mathbb{R}^{3}} f_{\varepsilon}(t, x, \xi) d \xi, \quad J_{\varepsilon}(t, x)=\int_{\mathbb{R}^{3}} \frac{1}{\sqrt{\varepsilon}} \xi f_{\varepsilon}(t, x, \xi) d \xi .
$$

The aim of this work is to investigate the effect of the friction forces, exerted mutually by the fluid and the particles, on the regularity and large time behavior of smooth solutions to (1.7)-(1.9).

The local existence of smooth solution is presented via an iterative method in Section 4. More precisely, we show that if the initial data are small in an appropriate norm, then the presence of friction forces which induce weak dissipation, can prevent the development of singularities and the Cauchy problem has a unique global smooth solution which decays in time at a rate.

Results on the global existence of smooth solutions near the constant state in Sobolev norms are established by the energy method. Decay rates of the $L^{p}$-norm of these solutions to the constant state are established when the $L^{q}$-norm of the perturbation is bounded. The details of this analysis is presented in [7].

The main strategy of our approach can be summarized as follows: We reformulate the Cauchy problem for (1.7)-(1.9) in such a way so that it is represented by the coupling of a symmetric hyperbolic system with a parabolic partial differential equation. The local existence of solutions to the symmetric hyperbolic system is obtained using the approach presented by Majda [35]. Next, an iterative method is presented for the construction of a sequence of approximate solutions for the Euler-Smoluchowski system. The local existence and finite propagation speed is presented in Section 4. 
The global existence of smooth solutions is established in [7] for the a class of forces which induce weak dissipation in the sense of Dafermos [22].

\section{Viscous model: global existence results}

We present in the sequel two notions of weak solutions to the Navier-StokesSmoluchowski system (1.1)-(1.3).

\subsection{Notions of weak solutions}

The following definition will be of use in the sequel.

Definition 2.1 (Confinement Hypotheses). Given a domain $\Omega \in C^{2, \nu}, \nu>$ $0, \Omega \subset \mathbb{R}^{3}$, and given a bounded-below external potential $\Phi: \Omega \longrightarrow \mathbb{R}_{0}^{+}$, the pair $(\Omega, \Phi)$ verifies the confinement hypotheses for the two-phase flow system (1.1)-(1.3) coupled with no-flux boundary conditions (1.4) whenever $\Phi$ is bounded and Lipschitz continuous in $\bar{\Omega}$ and the sub-level sets $\{\Phi<k\}$ are connected in $\Omega$ for any $k>0$. If $\Omega$ is unbounded, we assume that $\Phi \in W_{l o c}^{1, \infty}(\Omega), \beta>0$, the sub-level sets $[\Phi<k]$ are connected in $\Omega$ for any $k>0$,

$$
e^{-\Phi / 2} \in L^{1}(\Omega)
$$

and

$$
|\Delta \Phi(x)| \leq c_{1}\left|\nabla_{x} \Phi(x)\right| \leq c_{2} \Phi(x),|x|>R
$$

for some large $R>0$.

Let us now define a free-energy solution to the NSS system (1.1)-(1.3).

Definition 2.2 (Free-Energy Solution). Assume that $(\Omega, \Phi)$ satisfy the confinement hypotheses (HC). We say that $\{\varrho, \mathbf{u}, \eta\}$ is a free-energy solution of problem (1.1)-(1.3) supplemented with boundary data satisfying (1.4) and initial data $\left\{\varrho_{0}, \boldsymbol{m}_{0}, \eta_{0}\right\}$ satisfying (1.5) provided that the following hold:

- $\varrho \geq 0$ in $L^{\infty}\left(0, T ; L^{\gamma}(\Omega)\right)$ represents a renormalized solution of $(1.1)$ on $(0, \infty) \times \Omega$, i.e., for any test function $\phi \in \mathcal{D}([0, T) \times \bar{\Omega}), T>0$ and any $b, B$ such that

$$
b \in L^{\infty} \cap C[0, \infty), B(\varrho):=B(1)+\int_{1}^{\varrho} \frac{b(z)}{z^{2}} d z
$$


the renormalized continuity equation

$\int_{0}^{\infty} \int_{\Omega} B(\varrho) \partial_{t} \phi+B(\varrho) \mathbf{u} \cdot \nabla_{x} \phi-b(\varrho) \phi \operatorname{div}_{x} \mathbf{u} \mathrm{d} x \mathrm{~d} t=-\int_{\Omega} B\left(\varrho_{0}\right) \phi(0, \cdot) \mathrm{d} x$ holds.

- The balance of momentum (1.2) holds in the sense of distributions, i.e., for any $\mathbf{w} \in \mathcal{D}\left([0, T) ; \mathcal{D}\left(\bar{\Omega} ; \mathbb{R}^{3}\right)\right)$,

$$
\begin{aligned}
& \int_{0}^{\infty} \int_{\Omega} \varrho \mathbf{u} \cdot \partial_{t} \mathbf{w}+\varrho \mathbf{u} \otimes \mathbf{u}: \nabla_{x} \mathbf{w}+(p(\varrho)+\eta) \operatorname{div}_{x} \mathbf{w} \mathrm{d} x \mathrm{~d} t \\
& =\int_{0}^{\infty} \int_{\Omega} \mu \nabla_{x} \mathbf{u} \nabla_{x} \mathbf{w}+\lambda \operatorname{div}_{x} \mathbf{u} \operatorname{div}_{x} \mathbf{w}-(\beta \varrho+\eta) \nabla_{x} \Phi \cdot \mathbf{w} \mathrm{d} x \mathrm{~d} t \\
& -\int_{\Omega} \mathbf{m}_{0} \cdot \mathbf{w}(0, \cdot) \mathrm{d} x
\end{aligned}
$$

All quantities are required to be integrable, so in particular, $\mathbf{u} \in$ $L^{2}\left(0, T ; W^{1,2}\left(\Omega ; \mathbb{R}^{3}\right)\right)$, thus the velocity field can be required to vanish on $\partial \Omega$ in the sense of traces.

- $\eta \geq 0$ is a weak solution of (1.3), i.e.,

$\int_{0}^{\infty} \int_{\Omega} \eta \partial_{t} \phi+\eta \mathbf{u} \cdot \nabla_{x} \phi-\eta \nabla_{x} \Phi \cdot \nabla_{x} \phi-\nabla_{x} \eta \cdot \nabla_{x} \phi \mathrm{d} x \mathrm{~d} t=-\int_{\Omega} \eta_{0} \phi(0, \cdot) \mathrm{d} x$

Again, terms in this equation must be integrable on $(0, T) \times \Omega$, so in particular $\eta \in L^{2}\left(0, T ; L^{3}(\Omega)\right) \cap L^{1}\left(0, T ; W^{1, \frac{3}{2}}(\Omega)\right)$.

- The energy of the system

$$
\mathcal{F}(\varrho, \mathbf{u}, \eta)(\tau):=\int_{\Omega} \frac{1}{2} \varrho|\mathbf{u}|^{2}+\frac{a}{\gamma-1} \varrho^{\gamma}+\eta \ln \eta+(\beta \varrho+\eta) \Phi \mathrm{d} x(\tau)
$$

is finite and bounded by the initial energy. Also

$$
\int_{0}^{\infty} \int_{\Omega} \mu\left|\nabla_{x} \mathbf{u}\right|^{2}+\lambda\left|\operatorname{div}_{x} \mathbf{u}\right|^{2}+\left|2 \nabla_{x} \sqrt{\eta}+\sqrt{\eta} \nabla_{x} \Phi\right|^{2} \mathrm{~d} x \mathrm{~d} t \leq \mathcal{F}\left(\varrho_{0}, \mathbf{u}_{0}, \eta_{0}\right) .
$$

The following definition will be of use in the sequel.

Definition 2.3. In the spirit of Dafermos [19], given an entropy $\mathcal{E}(U)$, the relative entropy is defined as

$$
\mathcal{H}(U \mid \bar{U}):=\mathcal{E}(U)-\mathcal{E}(\bar{U})-D \mathcal{E}(\bar{U}) \cdot(U-\bar{U})
$$


where $D$ stands for the total differentiation operator with respect to $\varrho, \mathbf{m}$, and $\eta$.

In the present context,

$$
U=\left[\begin{array}{c}
\varrho \\
\mathbf{m}:=\varrho \mathbf{u} \\
\eta
\end{array}\right], \bar{U}=\left[\begin{array}{c}
r \\
\overline{\mathbf{m}}:=r \mathbf{U} \\
s
\end{array}\right]
$$

where $\bar{U}$ can be considered to be a smooth solution and

$$
\mathcal{E}(U):=\frac{|\mathbf{m}|^{2}}{2 \varrho}+\frac{a}{\gamma-1} \varrho^{\gamma}+\eta \ln \eta+(\beta \varrho+\eta) \Phi .
$$

Thus, from the definition, the relative entropy is

$$
\begin{aligned}
\mathcal{H}(U \mid \bar{U}) & =\frac{|\mathbf{m}|^{2}}{2 \varrho}+\frac{a}{\gamma-1} \varrho^{\gamma}+\eta \ln \eta+(\beta \varrho+\eta) \Phi \\
& -\frac{|\overline{\mathbf{m}}|^{2}}{2 r}-\frac{a}{\gamma-1} r^{\gamma}-s \ln s-(\beta r+s) \Phi \\
& -\left[\begin{array}{c}
-\frac{|\mathbf{U}|^{2}}{2}+\frac{a \gamma}{\gamma-1} r^{\gamma-1}+\beta \Phi \\
\mathbf{U} \\
\ln s+1+\Phi
\end{array}\right] \cdot\left[\begin{array}{c}
\varrho-r \\
\varrho \mathbf{u}-r \mathbf{U} \\
\eta-s
\end{array}\right] \\
& =\frac{\varrho|\mathbf{u}|^{2}}{2}+\frac{a}{\gamma-1} \varrho^{\gamma}+\eta \ln \eta+\beta \varrho \Phi+\eta \Phi \\
& -\frac{r|\mathbf{U}|^{2}}{2}-\frac{a}{\gamma-1} r^{\gamma}-s \ln s-\beta r \Phi-s \Phi \\
& +\frac{\varrho|\mathbf{U}|^{2}}{2}-\frac{r|\mathbf{U}|^{2}}{2}-\frac{a \gamma}{\gamma-1} r^{\gamma-1} \varrho+\frac{a \gamma}{\gamma-1} r^{\gamma}-\beta \varrho \Phi+\beta r \Phi \\
& -\varrho \mathbf{u} \cdot \mathbf{U}+r|\mathbf{U}|^{2}-\eta \ln s+s \ln s-\eta+s-\eta \Phi+s \Phi
\end{aligned}
$$

After some basic calculations, the relative entropy is calculated to be

$$
\begin{aligned}
\mathcal{H}(U \mid \bar{U})= & \frac{\varrho}{2}|\mathbf{u}-\mathbf{U}|^{2}+\frac{a}{\gamma-1}\left(\varrho^{\gamma}-r^{\gamma}\right)-\frac{a \gamma}{\gamma-1} r^{\gamma-1}(\varrho-r) \\
& +\eta \ln \eta-s \ln s-(\ln s+1)(\eta-s),
\end{aligned}
$$

or equivalently,

$$
\mathcal{H}(U \mid \bar{U})=\frac{\varrho}{2}|\mathbf{u}-\mathbf{U}|^{2}+E_{F}(\varrho, r)+E_{P}(\eta, s),
$$


where

$$
\begin{aligned}
& H_{F}(\varrho):=\frac{a}{\gamma-1} \varrho^{\gamma} \\
& P_{F}(\varrho):=H_{F}^{\prime}(\varrho)=\frac{a \gamma}{\gamma-1} \varrho^{\gamma-1} \\
& E_{F}(\varrho, r):=H_{F}(\varrho)-H_{F}^{\prime}(r)(\varrho-r)-H_{F}(r) \\
& H_{P}(\eta):=\eta \ln \eta \\
& P_{P}(\eta):=H_{P}^{\prime}(\eta)=\ln \eta+1 \\
& E_{P}(\eta, s):=H_{P}(\eta)-H_{P}^{\prime}(s)(\eta-s)-H_{P}(s)
\end{aligned}
$$

Remark 2.4. The integrals of the quantities $H_{F}$ and $H_{P}$ over $\Omega$ represent the physical quantities of the entropy of the fluid and the entropy of the particles, respectively.

Note that the relative entropy does not contain any information regarding the external potential $\Phi$. This is expected, as the relative entropy reflects information about quadratic terms, but not linear terms. Next, weakly dissipative solutions are defined using the ideas of relative entropy. The key addition to the definition of weak solutions is the requirement that the socalled relative entropy inequality is satisfied. Letting

$$
r=r(t, x), \mathbf{U}=\mathbf{U}(t, x), s=s(t, x)
$$

be smooth functions on $[0, T] \times \bar{\Omega}$ with $r, s>0$ on $[0, T] \times \bar{\Omega}$ and

$$
\left.\mathbf{U}\right|_{\partial \Omega}=0
$$

it is shown in $[6]$ that for smooth $\{\varrho, \mathbf{u}, \eta\}$,

$$
\begin{aligned}
& \int_{\Omega} \frac{1}{2} \varrho|\mathbf{u}-\mathbf{U}|^{2}+E_{F}(\varrho, r)+E_{P}(\eta, s) \mathrm{d} x(\tau) \\
& +\int_{0}^{\tau} \int_{\Omega}\left[\mathbb{S}\left(\nabla_{x} \mathbf{u}\right)-\mathbb{S}\left(\nabla_{x} \mathbf{U}\right)\right]: \nabla_{x}(\mathbf{u}-\mathbf{U}) \mathrm{d} x \mathrm{~d} t \\
& \leq \int_{\Omega} \frac{1}{2} \varrho_{0}\left|\mathbf{u}_{0}-\mathbf{U}_{0}\right|^{2}+E_{F}\left(\varrho_{0}, r_{0}\right)+E_{P}\left(\eta_{0}, s_{0}\right) \mathrm{d} x+\int_{0}^{\tau} \mathcal{R}(\varrho, \mathbf{u}, \eta, r, \mathbf{U}, s) \mathrm{d} t
\end{aligned}
$$

where

$$
\begin{aligned}
\mathcal{R} & (\varrho, \mathbf{u}, \eta, r, \mathbf{U}, s) \\
& :=\int_{\Omega} \operatorname{div}_{x}\left(\mathbb{S}\left(\nabla_{x} \mathbf{U}\right)\right) \cdot(\mathbf{U}-\mathbf{u}) \mathrm{d} x
\end{aligned}
$$




$$
\begin{aligned}
& -\int_{\Omega} \varrho\left(\partial_{t} \mathbf{U}+\mathbf{u} \cdot \nabla_{x} \mathbf{U}\right) \cdot(\mathbf{u}-\mathbf{U}) \mathrm{d} x \\
- & \int_{\Omega} \partial_{t} P_{F}(r)(\varrho-r)+\nabla_{x} P_{F}(r) \cdot(\varrho \mathbf{u}-r \mathbf{U}) \mathrm{d} x \\
- & \int_{\Omega}\left[\varrho\left(P_{F}(\varrho)-P_{F}(r)\right)-E_{F}(\varrho, r)\right] \operatorname{div}_{x} \mathbf{U} \mathrm{d} x \\
- & \int_{\Omega} \partial_{t} P_{P}(s)(\eta-s)+\nabla_{x} P_{P}(s) \cdot(\eta \mathbf{u}-s \mathbf{U}) \mathrm{d} x \\
- & \int_{\Omega}\left[\eta\left(P_{P}(\eta)-P_{P}(s)\right)-E_{P}(\eta, s)\right] \operatorname{div}_{x} \mathbf{U} \mathrm{d} x \\
- & \int_{\Omega} \nabla_{x}\left(P_{P}(\eta)-P_{P}(s)\right) \cdot\left(\nabla_{x} \eta+\eta \nabla_{x} \Phi\right) \mathrm{d} x \\
- & \int_{\Omega}(\beta \varrho+\eta) \nabla_{x} \Phi \cdot(\mathbf{u}-\mathbf{U}) \mathrm{d} x-\int_{\Omega} \frac{\eta \nabla_{x} s}{s} \cdot(\mathbf{u}-\mathbf{U}) \mathrm{d} x .
\end{aligned}
$$

Definition 2.5. We say that $\{\varrho, \mathbf{u}, \eta\}$ is a weakly dissipative solution of (1.1)-(1.3) with initial data $\left\{\varrho_{0}, \mathbf{u}_{0}, \eta_{0}\right\}$ if and only if

- $\{\varrho, \mathbf{u}, \eta\}$ is a free-energy solution on $(0, T)$ for some $T>0$ and that the energy inequality holds true

$$
\begin{aligned}
& \int_{\Omega} \frac{1}{2} \varrho|\mathbf{u}|^{2}+\frac{a}{\gamma-1} \varrho^{\gamma}+\eta \ln \eta+\eta \Phi \mathrm{d} x(\tau) \\
& +\int_{0}^{\tau} \int_{\Omega} \mathbb{S}\left(\nabla_{x} \mathbf{u}\right): \nabla_{x} \mathbf{u}+\left|2 \nabla_{x} \sqrt{\eta}+\sqrt{\eta} \nabla_{x} \Phi\right|^{2} \mathrm{~d} x \mathrm{~d} t \\
& \leq \int_{\Omega} \frac{1}{2} \varrho_{0}\left|\mathbf{u}_{0}\right|^{2}+\frac{a}{\gamma-1} \varrho_{0}^{\gamma}+\eta_{0} \ln \eta_{0}+\eta_{0} \Phi \mathrm{d} x \\
& -\beta \int_{0}^{\tau} \int_{\Omega} \varrho \mathbf{u} \cdot \nabla_{x} \Phi \mathrm{d} x \mathrm{~d} t \text { for } 0<\tau \leq T .
\end{aligned}
$$

- $\{\varrho, \mathbf{u}, \eta\}$ obeys inequality (2.10) for any suitably smooth functions $\{r, \mathbf{U}, s\}$.

From the work of [15], we have the following existence theorem for freeenergy solutions.

Theorem 2.6 (Global Existence of Free Energy Solutions). Assume that $(\Omega, \Phi)$ satisfy the confinement hypotheses. Then, the problem (1.1)-(1.3) supplemented with boundary conditions (1.4) and initial data satisfying (1.5) admits a weak solution $\{\varrho, \boldsymbol{u}, \eta\}$ on $(0, \infty) \times \Omega$ in the sense of Definition 2.2. In addition, 
i) the total fluid mass and particle mass given by

$$
M_{\varrho}(t)=\int_{\Omega} \varrho(t, \cdot) d x \quad \text { and } \quad M_{\eta}(t)=\int_{\Omega} \eta(t, \cdot) d x,
$$

respectively, are constants of motion.

ii) the density satisfies the higher integrability result

$$
\varrho \in L^{\gamma+\Theta}((0, T) \times \Omega),
$$

where $\Theta=\min \left\{\frac{2}{N} \gamma-1, \frac{\gamma}{3}\right\}$.

Proof. The main ingredients in the proof of this result can be formulated as follows:

- A suitable weak formulation of the underlying physical principles is introduced. Physically grounded hypotheses are imposed on the system as follows. The mixture occupies the physical space $\Omega \subset \mathbb{R}^{3}$. The boundary conditions are chosen in such a way that the dissipation of energy is guaranteed.

- A priori estimates are established, based solely on the boundedness of the initial energy and entropy of the system.

- A suitable approximating scheme is introduced for the construction of the solution based on a two-level approximating procedure: the first level involves an artificial pressure approximation, whereas the second-level approximation employs a time-discretization scheme. The sequence of approximate solutions is constructed with the aid of a fixed point argument coupling the time discretized compressible isentropic Navier-Stokes equations to a discretization in time of the equation for $\eta$.

- Physically grounded hypotheses are imposed on the domain $\Omega$ and the external potential $\Phi$ (confinement hypotheses (HC)). The analysis in the present article treats both the case of a bounded physical domain and the case of an unbounded domain. We remark that the confinement hypothesis $(\mathbf{H C})$ on $(\Omega, \Phi)$ plays a crucial role in providing control of the negative contribution of the physical entropy $\eta \ln \eta$ in the free energy bounds for unbounded domains For details, the reader may consult the work in [15].

Theorem 2.7 (Global Existence of Weakly Dissipative Solutions). Assume that $(\Omega, \Phi)$ satisfy the confinement hypotheses with $\Omega \subset \mathbb{R}^{3}$ a bounded domain of class $C^{2, \nu}, \nu>0$. Suppose the initial data $\left\{\varrho_{0}, \boldsymbol{u}_{0}, \eta_{0}\right\}$ satisfy

$\varrho_{0}$ not identically zero, $\varrho_{0}\left|\boldsymbol{u}_{0}\right|^{2} \in L^{1}(\Omega)$, and $\eta_{0} \ln \eta_{0} \in L^{1}(\Omega)$ 
in addition to the conditions on the initial data specified in (1.5). Then the Navier-Stokes-Smoluchowski system in (1.1)-(1.3) has a weakly dissipative solution.

Proof. An inherent definition of weakly dissipative solutions to the NavierStokes Smoluchowski system (1.1)-(1.3) is introduced satisfying a relative entropy inequality with respect to any hypothetical strong solution to the problem. The global existence of weakly dissipative solutions is established by the construction of a suitable approximating scheme. Convergence arguments are employed to show that the solutions to the actual system obey a relative entropy inequality. For the details of the analysis we refer the reader to $[6]$.

\subsection{Weak-strong uniqueness result}

First, we assume that we have a smooth solution $\{r, \mathbf{U}, s\}$ such that

$$
\begin{aligned}
& r \in C_{\text {weak }}\left([0, T] ; L^{\gamma}(\Omega)\right) \\
& \mathbf{U} \in C_{\text {weak }}\left([0, T] ; L^{2 \gamma / \gamma-1}\left(\Omega ; \mathbb{R}^{3}\right)\right) \\
& \nabla_{x} \mathbf{U} \in L^{2}\left(0, T ; L^{2}\left(\Omega ; \mathbb{R}^{3 \times 3}\right)\right),\left.\mathbf{U}\right|_{\partial \Omega}=0 \\
& s \in C_{\text {weak }}\left([0, T] ; L^{1}(\Omega)\right) \cap L^{1}\left(0, T ; L^{6 \gamma / \gamma-3}(\Omega)\right) \\
& \partial_{t} \mathbf{U} \in L^{1}\left(0, T ; L^{2 \gamma / \gamma-1}\left(\Omega ; \mathbb{R}^{3}\right)\right) \cap L^{2}\left(0, T ; L^{6 \gamma / 5 \gamma-6}\left(\Omega ; \mathbb{R}^{3}\right)\right) \\
& \nabla_{x}^{2} \mathbf{U} \in L^{1}\left(0, T ; L^{2 \gamma / \gamma+1}\left(\Omega ; \mathbb{R}^{3 \times 3 \times 3}\right)\right) \cap L^{2}\left(0, T ; L^{6 \gamma / 5 \gamma-6}\left(\Omega ; \mathbb{R}^{3 \times 3 \times 3}\right)\right) \\
& \partial_{t} P_{F}(r) \in L^{1}\left(0, T ; L^{\gamma / \gamma-1}(\Omega)\right) \\
& \nabla_{x} P_{F}(r) \in L^{1}\left(0, T ; L^{2 \gamma / \gamma-1}\left(\Omega ; \mathbb{R}^{3}\right)\right) \cap L^{2}\left(0, T ; L^{6 \gamma / 5 \gamma-6}\left(\Omega ; \mathbb{R}^{3}\right)\right) \\
& \partial_{t} P_{P}(s) \in L^{1}\left(0, T ; L^{\infty}(\Omega)\right) \cap L^{\infty}\left(0, T ; L^{3 / 2}(\Omega)\right) \\
& \nabla_{x} P_{P}(s) \in L^{\infty}\left(0, T ; L^{3}\left(\Omega ; \mathbb{R}^{3}\right)\right) \\
& \nabla_{x} s \in L^{\infty}\left(0, T ; L^{2}\left(\Omega ; \mathbb{R}^{3}\right)\right) \cap L^{2}\left(0, T ; L^{6 \gamma / 5 \gamma+3}\left(\Omega ; \mathbb{R}^{3}\right)\right) .
\end{aligned}
$$

The quantity $\{r, \mathbf{U}, s\}$ is taken to be a solution of (1.1)-(1.4) with the regularity stated above and $r$ and $s$ are taken to be bounded above and bounded below by some positive constant. Also, $\mathbf{U}$ is taken to be bounded above in magnitude and the following conditions are imposed:

$$
\begin{aligned}
& \nabla_{x} r \in L^{2}\left(0, T ; L^{q}\left(\Omega ; \mathbb{R}^{3}\right)\right) \\
& \nabla_{x}^{2} \mathbf{U} \in L^{2}\left(0, T ; L^{q}\left(\Omega ; \mathbb{R}^{3 \times 3 \times 3}\right)\right) \\
& \alpha:=\nabla_{x} s+s \nabla_{x} \Phi \in L^{2}\left(0, T ; L^{q}\left(\Omega ; \mathbb{R}^{3}\right)\right)
\end{aligned}
$$


where

$$
q>\max \left\{3, \frac{3}{\gamma-1}\right\} .
$$

Thus, by embeddings, $\mathbf{U} \in L^{2}\left(0, T ; W^{1, \infty}\left(\Omega ; \mathbb{R}^{3}\right)\right)$ since $\gamma>\frac{3}{2}, q>\frac{6 \gamma}{5 \gamma-6}$. Now the weak-strong uniqueness result is stated:

Theorem 2.8 (Weak-Strong Uniqueness). Assume $\{\varrho, \boldsymbol{u}, \eta\}$ is a weakly dissipative solution of (1.1)-(1.4). Assume that $\{r, \boldsymbol{U}, s\}$ is a smooth solution of (1.1)-(1.4) with the regularity stated above and obeying the hypotheses (2.13)-(2.15). Then $\{\varrho, \boldsymbol{u}, \eta\}$ is identically $\{r, \boldsymbol{U}, s\}$.

Proof. The proof of this theorem is provided by Ballew and Trivisa in [6]. The method employed in this work is somewhat reminiscent of the relative entropy method of Dafermos [19], DiPerna [21]. We refer the reader to the articles by Berthelin and Vasseur [11], Mellet and Vasseur [36], where this method was employed in different settings.

Remark 2.9. This result can be extended to unbounded domains by considering a collection of bounded subsets and taking the limit using the confinement hypotheses. See [6] for details.

Remark 2.10. This theorem does not show the existence of smooth solutions $\{r, \mathbf{U}, s\}$. For such a regularity result, the reader may consult [3].

\section{Viscous model: asymptotic analysis}

In this section, we present some results on asymptotic behavior of solutions to the NSS system. First, we show formally that free-energy solutions converge to solutions to the stationary NSS system as $t \rightarrow \infty$. Next, we show formal results for low Mach number limits to the NSS system and state the main theorems proven in [5].

3.0.1. Convergence to a stationary solution In this section we see what happens if we let $t \rightarrow \infty$ in the free-energy solutions of the NSS system. Formally, we can consider the total energy $\mathcal{F}$ to be a Lyapunov functional satisfying

$$
\frac{\mathrm{d} \mathcal{F}}{\mathrm{d} t}+\int_{\Omega} \mu\left|\nabla_{x} \mathbf{u}\right|^{2}+\lambda\left|\operatorname{div}_{x} \mathbf{u}\right|^{2}+\left|2 \nabla_{x} \sqrt{\eta}+\sqrt{\eta} \nabla_{x} \Phi\right|^{2} \mathrm{~d} x \leq 0 .
$$

From (3.1), we see formaly that as $t \rightarrow \infty$ then $\mathbf{u} \rightarrow 0$. Thus, (1.2) becomes

$$
\nabla_{x}\left(p_{F}(\varrho)+\eta\right)=-(\beta \varrho+\eta) \nabla_{x} \Phi
$$


But writing the energy estimate as

$$
\begin{aligned}
& \mathcal{F}(T)+\int_{0}^{T} \mu\left\|\nabla_{x} \mathbf{u}(t)\right\|_{L^{2}\left(\Omega ; \mathbb{R}^{3 \times 3}\right)}^{2}+\lambda\left\|\operatorname{div}_{x} \mathbf{u}(t)\right\|_{L^{2}(\Omega)}^{2} \mathrm{~d} t \\
& +\int_{0}^{T} \int_{\Omega}\left|2 \nabla_{x} \sqrt{\eta}+\sqrt{\eta} \nabla_{x} \Phi\right|^{2} \mathrm{~d} x \mathrm{~d} t \leq \mathcal{F}(0)
\end{aligned}
$$

we get

$$
\left|2 \nabla_{x} \sqrt{\eta}+\sqrt{\eta} \nabla_{x} \Phi\right|^{2}=0
$$

So, defining

$$
\begin{aligned}
& \varrho_{n}:=\varrho\left(t+\tau_{n}, \cdot\right) \\
& \eta_{n}:=\eta\left(t+\tau_{n}, \cdot\right)
\end{aligned}
$$

where $\left\{\tau_{n}\right\}$ is a sequence such that $\tau_{n} \rightarrow \infty$, we have $\varrho_{n} \rightarrow \varrho_{s}$ and $\eta_{n} \rightarrow \eta_{s}$ where

$$
\begin{aligned}
& \nabla_{x} p_{F}\left(\varrho_{s}\right)=-\beta \varrho_{s} \nabla_{x} \Phi \\
& \nabla_{x} \eta_{s}=-\eta_{s} \nabla_{x} \Phi .
\end{aligned}
$$

Indeed, we have the following theorem which is proven rigorously in Section 4 of $[15]$.

Theorem 3.1 (Large-time Asymptotics). Let us assume that $(\Omega, \Phi)$ satisfy the confinement hypotheses. Then, for any free-energy solution $(\varrho, \boldsymbol{u}, \eta)$ of the problem $(1.1)-(1.3)$, there exist universal stationary states $\varrho_{s}=\varrho_{s}(x)$, $\eta_{s}=\eta_{s}(x)$, such that

$$
\left\{\begin{array}{l}
\varrho(t) \rightarrow \varrho_{s} \text { strongly in } L^{\gamma}(\Omega) \\
\text { ess } \sup _{\tau>t} \int_{\Omega} \varrho(\tau)|\boldsymbol{u}(\tau)|^{2} d x \rightarrow 0 \\
\eta(t) \rightarrow \eta_{s} \text { strongly in } L^{p_{2}}(\Omega) \text { for } p_{2}>1
\end{array}\right.
$$

as $t \rightarrow \infty$, where $\left(\eta_{s}, \varrho_{s}\right)$ are characterized as the unique free-energy solution of the stationary state problem:

$$
\begin{aligned}
& \nabla_{x} p_{F}\left(\varrho_{s}\right)=-\beta \varrho_{s} \nabla_{x} \Phi \\
& \nabla_{x} \eta_{s}=-\eta_{s} \nabla_{x} \Phi
\end{aligned}
$$


Proof. The key ingredient in the proof of this result is the establishment of the appropriate compactness which allows us to take the limit $\tau_{n} \rightarrow \infty$ in the sequences:

$$
\begin{aligned}
& \varrho_{n}:=\varrho\left(t+\tau_{n}, \cdot\right) \\
& \eta_{n}:=\eta\left(t+\tau_{n}, \cdot\right)
\end{aligned}
$$

to establish that $\varrho_{n} \rightarrow \varrho_{s}$ and $\eta_{n} \rightarrow \eta_{s}$. The strong convergence of the density sequences $\left\{\varrho_{n}\right\}$ is obtained by showing the weak continuity of the effective viscous pressure. The strong convergence of the particle density sequence $\left\{\eta_{n}\right\}$ is obtained using the Lions-Aubin Lemma.

\subsection{Low stratification low mach number limit}

Before we get to the formal calculations for the low stratification limit, we present the scaling of the NSS system. We start by adding the values $D$, describing the dispersion of the particles in the fluid, and $\zeta$, a drag coefficient, to ensure consistency of the physical units in the equations. Specifically, the pressure term in the momentum equation becomes

$$
\nabla_{x}\left(a \varrho^{\gamma}+\frac{D}{\zeta} \eta\right)
$$

the Smoluchowski equation becomes

$$
\partial_{t} \eta+\operatorname{div}_{x}(\eta \mathbf{u})-\operatorname{div}_{x}(\zeta \eta \Phi)-D \Delta_{x} \eta=0,
$$

and the energy inequality becomes

$$
\begin{gathered}
\int_{\Omega} \frac{1}{2} \varrho|\mathbf{u}|^{2}+\frac{a}{\gamma-1} \varrho^{\gamma}+\frac{D}{\zeta} \eta \ln \eta+(\beta \varrho+\eta) \Phi \mathrm{d} x(\tau) \\
+\int_{0}^{\infty} \int_{\Omega} \mu\left|\nabla_{x} \mathbf{u}\right|^{2}+\lambda\left|\operatorname{div}_{x} \mathbf{u}\right|^{2}+\left|2 \nabla_{x} \frac{D}{\sqrt{\zeta}} \sqrt{\eta}+\sqrt{\zeta \eta} \nabla_{x} \Phi\right|^{2} \mathrm{~d} x \mathrm{~d} t \\
\leq \int_{\Omega} \frac{1}{2} \varrho_{0}\left|\mathbf{u}_{0}\right|^{2}+\frac{a}{\gamma-1} \varrho_{0}^{\gamma}+\frac{D}{\zeta} \eta_{0} \ln \eta_{0}+\left(\beta \varrho_{0}+\eta_{0}\right) \Phi \mathrm{d} x .
\end{gathered}
$$

To begin the scaling of the Navier-Stokes-Smoluchowski model, the quantities

$$
\varrho, \mathbf{u}, \eta, \zeta, D, p_{F}, p_{P}, \Phi, \mu \text {, and } \lambda,
$$

where $p_{P}(\eta):=\frac{D}{\zeta} \eta$, as well as the time and length scales, must be made nondimensional. This is done by defining for each quantity $A$ a reference value 
$A_{r e f}$ which also reflects the physical unit of measurement for that quantity, such as meter, second, meter per second, et cetera. Then, the dimensionless value $A^{\prime}$ is defined as

$$
A^{\prime}:=\frac{A}{A_{\text {ref }}} .
$$

After applying the chain rule and some straight-forward algebra, the formal dimensionless Navier-Stokes-Smoluchowski system becomes (omitting the primes for the sake of notational simplicity)

$$
\begin{gathered}
\operatorname{Sr}_{t} \varrho+\operatorname{div}_{x}(\varrho \mathbf{u})=0 \\
\operatorname{Sr}_{t}(\varrho \mathbf{u})+\operatorname{div}_{x}(\varrho \mathbf{u} \otimes \mathbf{u})+\frac{1}{\mathrm{Ma}^{2}} \nabla_{x}\left(a \varrho^{\gamma}+\operatorname{Pc} \frac{D}{\zeta} \eta\right) \\
=\frac{1}{\operatorname{Re}}\left(\mu \Delta_{x} \mathbf{u}+\lambda \nabla_{x} \operatorname{div}_{x} \mathbf{u}\right)-\frac{1}{\operatorname{Fr}^{2}}(\beta \varrho+\operatorname{Dc} \eta) \nabla_{x} \Phi \\
\operatorname{Sr}_{t} \eta+\operatorname{div}_{x}(\eta \mathbf{u})-\operatorname{Za}_{\operatorname{div}_{x}}\left(\zeta \eta \nabla_{x} \Phi\right)-\operatorname{Da} D \Delta_{x} \eta=0
\end{gathered}
$$

The total energy inequality for the scaled system takes the form.

$$
\begin{aligned}
& \operatorname{Sr} \frac{\mathrm{d}}{\mathrm{d} t} \int_{\Omega} \frac{\mathrm{Ma}^{2}}{2} \varrho|\mathbf{u}|^{2}+\frac{a}{\gamma-1} \varrho^{\gamma}+\operatorname{Pc} \frac{D \eta}{\zeta} \ln \eta+\frac{\mathrm{Ma}^{2}}{\mathrm{Fr}^{2}}(\beta \varrho+\mathrm{Dc} \eta) \Phi \mathrm{d} x \\
& +\int_{\Omega} \frac{\mathrm{Ma}^{2}}{\operatorname{Re}} \mathbb{S}\left(\nabla_{x} \mathbf{u}\right): \nabla_{x} \mathbf{u} \mathrm{d} x \\
& +\int_{\Omega} \operatorname{PcDa} D^{2} \frac{\left|\nabla_{x} \eta\right|^{2}}{\zeta \eta}+2 \mathrm{Za} D \nabla_{x} \eta \cdot \nabla_{x} \Phi+\frac{\mathrm{Za}^{2}}{\mathrm{Da}} \zeta \eta\left|\nabla_{x} \Phi\right|^{2} \mathrm{~d} x \leq 0 .
\end{aligned}
$$

Here, the non-dimensional parameters used in (3.8)-(3.11) are defined as follows:

Table 3.1: Definitions of the Dimensionless Parameters

$$
\begin{array}{ccc}
\text { Sr: }:=\frac{L_{r e f}}{\mathbf{u}_{r e f} t_{r e f}} & \text { Ma: }=\frac{\mathbf{u}_{r e f}}{\sqrt{p_{F_{r e f}} / \varrho_{r e f}}} & \text { Re: }:=\frac{\varrho_{r e f} \mathbf{u}_{r e f} L_{r e f}}{\mu_{r e f}} \\
\text { Fr: }:=\frac{\mathbf{u}_{r e f}}{\sqrt{L_{r e f} f_{r e f}}} & \text { Za: }:=\frac{\zeta_{r e f} f_{r e f}}{\mathbf{u}_{r e f}} & \text { Da: }:=\frac{D_{r e f}}{L_{r e f} \mathbf{u}_{r e f}} \\
\text { Pc }:=\frac{p_{P_{r e f}}}{p_{F_{r e f}}} & \text { Dc: }:=\frac{\eta_{r e f}}{\varrho_{r e f}} . &
\end{array}
$$

where the quantities

$$
L_{r e f}, \mathbf{u}_{r e f}, t_{r e f}, p_{F_{r e f}}, \varrho_{r e f}, \mu_{r e f}, f_{r e f}, \zeta_{r e f}, D_{r e f}, p_{P_{r e f}}, \text { and } \eta_{r e f}
$$


represent the reference values for the length, velocity, time, fluid pressure, fluid density, viscosity coefficient, force (equal to $\nabla_{x} \Phi$ ), drag coefficient, diffusivity coefficient, particle pressure, and particle density, respectively. The compatibility conditions $\mu_{r e f}=\lambda_{r e f}$ and $p_{F_{r e f}}=\varrho_{r e f} e_{F_{r e f}}, p_{P_{r e f}}=$ $\eta_{\text {ref }} e_{P_{\text {ref }}}$ are also imposed to obtained the scaling, the second and third of which follow naturally from Maxwell's relation. Note also that Ma represents the Mach number, Sr the Strouhal number, Re the Reynolds number, and Fr the Froude number. Since existence of solutions to the scaled system follows from [6] and [15] for any choices of positive values of the dimensionless parameters, various singular limits can be explored. The current section considers a low-Mach-number limit, with Ma taken to be a small parameter $\varepsilon$, Za scaled as Ma, and $\mathrm{Fr}=\sqrt{\varepsilon}$.

$$
\begin{gathered}
\partial_{t} \varrho_{\varepsilon}+\operatorname{div}_{x}\left(\varrho_{\varepsilon} \mathbf{u}_{\varepsilon}\right)=0 \\
\varepsilon^{2}\left[\partial_{t}\left(\varrho_{\varepsilon} \mathbf{u}_{\varepsilon}\right)+\operatorname{div}_{x}\left(\varrho_{\varepsilon} \mathbf{u}_{\varepsilon} \otimes \mathbf{u}_{\varepsilon}\right)\right]+\nabla_{x}\left(a \varrho_{\varepsilon}^{\gamma}+\frac{D}{\zeta} \eta_{\varepsilon}\right) \\
=\varepsilon^{2}\left(\mu \Delta_{x} \mathbf{u}_{\varepsilon}+\lambda \nabla_{x} \operatorname{div}_{x} \mathbf{u}_{\varepsilon}\right)-\varepsilon\left(\beta \varrho_{\varepsilon}+\eta_{\varepsilon}\right) \nabla_{x} \Phi \\
\partial_{t} \eta_{\varepsilon}+\operatorname{div}_{x}\left(\eta_{\varepsilon} \mathbf{u}_{\varepsilon}\right)-\varepsilon \operatorname{div}_{x}\left(\zeta \eta_{\varepsilon} \nabla_{x} \Phi\right)-D \Delta_{x} \eta_{\varepsilon}=0 \\
\int_{\Omega} \frac{\varepsilon^{2}}{2} \varrho_{\varepsilon}\left|\mathbf{u}_{\varepsilon}\right|^{2}+\frac{a}{\gamma-1} \varrho_{\varepsilon}^{\gamma}+\frac{D \eta_{\varepsilon}}{\zeta} \ln \eta_{\varepsilon}+\varepsilon\left(\beta \varrho_{\varepsilon}+\eta_{\varepsilon}\right) \Phi \mathrm{d} x(T) \\
+\int_{0}^{\infty} \int_{\Omega} \varepsilon^{2}\left(\mu\left|\nabla_{x} \mathbf{u}_{\varepsilon}\right|^{2}+\lambda\left|\operatorname{div} \mathbf{u}_{\varepsilon}\right|^{2} \mathrm{~d} x \mathrm{~d} t\right. \\
+\int_{0}^{\infty} \int_{\Omega}\left|2 \frac{D}{\sqrt{\zeta}} \nabla_{x} \sqrt{\eta_{\varepsilon}}+\varepsilon \sqrt{\zeta \eta_{\varepsilon}} \nabla_{x} \Phi\right|^{2} \mathrm{~d} x \mathrm{~d} t \\
\leq \int_{\Omega} \frac{\varepsilon^{2}}{2} \varrho_{0}\left|\mathbf{u}_{0}\right|^{2}+\frac{a}{\gamma-1} \varrho_{0}^{\gamma}+\frac{D \eta_{0}}{\zeta} \ln \eta_{0}+\varepsilon\left(\beta \varrho_{0}+\eta_{0}\right) \Phi \mathrm{d} x
\end{gathered}
$$

The formal approach is to expand $\varrho_{\varepsilon}, \mathbf{u}_{\varepsilon}$, and $\eta_{\varepsilon}$ as

$$
\begin{aligned}
& \varrho_{\varepsilon}=\bar{\varrho}+\sum_{i=1}^{\infty} \varepsilon^{i} \varrho^{(i)} \\
& \mathbf{u}_{\varepsilon}=\overline{\mathbf{u}}+\sum_{i=1}^{\infty} \varepsilon^{i} \mathbf{u}^{(i)} \\
& \eta_{\varepsilon}=\bar{\eta}+\sum_{i=1}^{\infty} \varepsilon^{i} \eta^{(i)}
\end{aligned}
$$


plug these expansions into (3.12)-(3.15), and equate terms of equal orders of $\varepsilon$. In doing so, it becomes clear that since the right side of (3.15) is bounded uniformly in $\varepsilon$, as it is just the initial energy, it must be true that

$$
\nabla_{x} \sqrt{\bar{\eta}}=0 .
$$

Thus, $\bar{\eta}$ is constant on $\Omega$ for each time $t$ and

$$
\bar{\eta}=\frac{1}{|\Omega|} \int_{\Omega} \eta_{0} d x
$$

in the formal limit. Moving to the momentum equation (3.13) and equating terms of order one, the formal equation becomes

$$
\nabla_{x}\left(a \bar{\varrho}^{\gamma}+\frac{D}{\zeta} \bar{\eta}\right)=0 .
$$

Since $\bar{\eta}$ is constant, it follows formally that

$$
\bar{\varrho}=\frac{1}{|\Omega|} \int_{\Omega} \varrho_{0} d x .
$$

Using this fact in the continuity equation (3.12) and equating terms of order one yields the incompressibility condition for the limit velocity

$$
\operatorname{div}_{x} \overline{\mathbf{u}}=0 .
$$

Returning to (3.15) and equating terms of order $\varepsilon^{2}$, it is easy to show formally that

$$
\bar{\varrho}\left[\partial_{t} \overline{\mathbf{u}}+\operatorname{div}_{x}(\overline{\mathbf{u}} \otimes \overline{\mathbf{u}})\right]+\nabla_{x} \Pi=\mu \Delta_{x} \overline{\mathbf{u}}-(\beta r+\theta) \nabla_{x} \Phi
$$

where $r, \theta$ are related to the limit quantities by

$$
\nabla_{x}\left(a r^{\gamma}+\frac{D}{\zeta} \theta\right)=-(\beta \bar{\varrho}+\bar{\eta}) \nabla_{x} \Phi
$$

which is found by equating terms of order $\varepsilon$ in (3.13) and relabeling $\varrho^{(1)}$ and $\eta^{(1)}$. Thus, the formal low stratification low Mach number limit for the Navier-Stokes-Smoluchowski system becomes

$$
\bar{\eta}=\frac{1}{|\Omega|} \int_{\Omega} \eta_{0}(x) \mathrm{d} x
$$




$$
\begin{aligned}
& \bar{\varrho}=\frac{1}{|\Omega|} \int_{\Omega} \varrho_{0}(x) \mathrm{d} x \\
& \operatorname{div}_{x} \overline{\mathbf{u}}=0 \\
& \varrho\left[\partial_{t} \overline{\mathbf{u}}+\operatorname{div}_{x}(\overline{\mathbf{u}} \otimes \overline{\mathbf{u}})\right]+\nabla_{x} \Pi=\mu \Delta_{x} \overline{\mathbf{u}}-(\beta r+\theta) \nabla_{x} \Phi
\end{aligned}
$$

where $r, \theta$ satisfy

$$
\nabla_{x}\left(a r^{\gamma}+\frac{D}{\zeta} \theta\right)=-(\beta \bar{\varrho}+\bar{\eta}) \nabla_{x} \Phi
$$

and $\Pi$ is a function incorporating the terms for which a gradient is taken.

We now introduce a geometric condition on $\Omega$ which plays a crucial role in the study of propagation of the acoustic waves. Let us consider the following problem:

$$
-\Delta \phi=\lambda \phi \quad \text { in } \Omega, \quad \frac{\partial \phi}{\partial \mathbf{n}}=0 \text { on } \partial \Omega,
$$

where $\phi$ is constant on $\partial \Omega$. We call a solution of the problem (3.21) trivial if $\lambda=0$ and $\phi$ is constant.

Definition 3.2. We say that $\Omega$ verifies assumption $(\mathrm{H})$ if all solutions of the problem (3.21) are trivial.

Remark 3.3. Notice that Schiffer's conjecture shows that every $\Omega$ satisfies (H) except the ball and Feireisl, Novotny, Petzeltova [27] gives an example of domain $\Omega$ which is trivial. In two dimensional space, it is proven that every bounded, simply connected open domain $\Omega \subset \mathbb{R}^{2}$ whose boundary id Lipschitz but not real analytic satisfies $(\mathrm{H})$.

This formal analysis leads to the following theorem, which is proven rigorously in [5].

Theorem 3.4 (Low stratification limit). Let $\Omega \subset \mathbb{R}^{3}$ be a bounded domain with a boundary of class $C^{2+\nu}, \nu>0$ verifying the geometric condition $(H)$. Let $(\Omega, \Phi)$ satisfy the confinement hypothesis and assume $Z a=\mathrm{Ma}=\varepsilon$, $\mathrm{Fr}=\sqrt{\varepsilon}$ and $\left\{\varrho_{\varepsilon}, \boldsymbol{u}_{\varepsilon}, \eta_{\varepsilon}\right\}_{\varepsilon>0}$ is a family of free-energy solutions to the scaled Navier-Stokes Smoluchowski system with the boundary conditions

$$
\left.\boldsymbol{u}\right|_{\partial \Omega}=\left.\left(\varepsilon \eta_{\varepsilon} \nabla_{x} \Phi+\nabla_{x} \eta_{\varepsilon}\right) \cdot \boldsymbol{n}\right|_{\partial \Omega}=0
$$

Assume the initial condition as follows.

$$
\varrho_{\varepsilon}(0, \cdot)=\varrho_{\varepsilon, 0}=\bar{\varrho}+\varepsilon \varrho_{\varepsilon, 0}^{(1)}, \boldsymbol{u}_{\varepsilon}(0, \cdot)=\boldsymbol{u}_{\varepsilon, 0}, \eta(0, \cdot)=\bar{\eta}+\varepsilon \eta_{\varepsilon, 0}^{(1)}
$$


where

$$
\bar{\varrho}=\frac{1}{|\Omega|} \int_{\Omega} \varrho_{\varepsilon, 0} d x, \bar{\eta}=\frac{1}{|\Omega|} \int_{\Omega} \eta_{\varepsilon, 0} d x
$$

and

$$
\varrho_{\varepsilon, 0} \rightarrow \varrho_{0}^{(1)}, \boldsymbol{u}_{\varepsilon, 0} \rightarrow \boldsymbol{u}_{0}^{(1)}, \eta_{\varepsilon, 0} \rightarrow \eta_{0}^{(1)},
$$

as $\varepsilon$ tends to 0 using weak-* convergence in $L^{\infty}(\Omega)$. Then, up to subsequences,

$$
\left\{\begin{array}{l}
\varrho_{\epsilon} \rightarrow \bar{\varrho} \text { in } C\left([0, T] ; L^{1}(\Omega)\right) \cap L^{\infty}\left(0, T ; L^{\frac{5}{3}}(\Omega)\right) \\
\eta_{\epsilon} \rightarrow \bar{\eta} \text { in } L^{2}\left(0, T ; W^{1,2}(\Omega)\right) \\
\boldsymbol{u}_{\epsilon} \rightarrow \overline{\boldsymbol{u}} \text { strongly in } L^{2}\left(0, T ; L^{2}\left(\Omega ; \mathbb{R}^{3}\right)\right)
\end{array}\right.
$$

and

$$
\left\{\begin{array}{l}
\varrho_{\varepsilon}^{(1)}=\frac{\varrho_{\varepsilon}-\bar{\varrho}}{\varepsilon} \rightarrow \varrho^{(1)} \text { weakly }-* \text { in } L^{\infty}\left(0, T ; L^{q}(\Omega)\right),, q=\min \{2, \gamma\} \\
\eta_{\varepsilon}^{(1)}=\frac{\eta_{\varepsilon}-\bar{\eta}}{\varepsilon} \rightarrow \eta^{(1)} \text { weakly in } L^{2}\left(0, T ; L^{2}(\Omega)\right)
\end{array}\right.
$$

where $\left\{\overline{\boldsymbol{u}}, r=\varrho^{(1)}, \theta=\eta^{(1)}\right\}$ solve the target system (3.16)-(3.20) weakly with the boundary condition $\left.\boldsymbol{u}\right|_{\partial \Omega}=0$ and the initial data

$$
\boldsymbol{u}(0)=\boldsymbol{H}\left[\boldsymbol{u}_{0}\right]
$$

where the Helmholtz's projection $\boldsymbol{H}$ is defined by

$$
\boldsymbol{H}=\boldsymbol{I}-\boldsymbol{H}^{\perp}, \boldsymbol{H}^{\perp}=\nabla_{x} \Delta_{x}^{-1} \operatorname{div}_{x} .
$$

Proof. Our strategy can be summarized as follows. First, using the free energy inequality, uniform bounds in $\varepsilon$ are obtained. Using these bounds convergence results on some relevant quantities are established. In order to establish rigorously the limits of the sequence of solution $\left\{\varrho_{\varepsilon}, \mathbf{u}_{\varepsilon}, \eta_{\varepsilon}\right\}_{\varepsilon>0}$ we need to show that the divergence of the term $\overline{\varrho \mathbf{u}} \otimes \overline{\mathbf{u}}-\overline{\varrho \mathbf{u} \otimes \mathbf{u}}$ converges weakly to a gradient. This can be established by employing the standard Helmholtz decomposition in order to decompose this quantity into a divergence-free and a gradient part. For the details we refer the reader to $[5]$. 


\subsection{Strong stratification and low mach number limit}

The next limit considered here is a strong stratification limit. In this case, the Froude number is scaled the same as the Mach number limit, and the values are scaled as stated below:

- Ma is taken to be a small parameter $\varepsilon>0$.

- Za, Da are taken to be $\varepsilon^{-1}$.

- Fr is taken to be $\varepsilon$.

- Other parameters are taken to be of order 1.

- The external potential takes the form $\Phi=g x_{3}$ where $g$ is a constant (gravity/buoyancy).

Thus, the scaled NSS system becomes

(3.29) $\partial_{t} \varrho_{\varepsilon}+\operatorname{div}_{x}\left(\varrho_{\varepsilon} \mathbf{u}_{\varepsilon}\right)=0$

(3.30) $\varepsilon^{2}\left[\partial_{t}\left(\varrho_{\varepsilon} \mathbf{u}_{\varepsilon}\right)+\operatorname{div}_{x}\left(\varrho_{\varepsilon} \mathbf{u}_{\varepsilon} \otimes \mathbf{u}_{\varepsilon}\right)\right]+\nabla_{x}\left(a \varrho_{\varepsilon}^{\gamma}+\frac{D}{\zeta} \eta_{\varepsilon}\right)$

$$
=\varepsilon^{2}\left(\mu \Delta_{x} \mathbf{u}_{\varepsilon}+\lambda \nabla_{x} \operatorname{div}_{x} \mathbf{u}_{\varepsilon}\right)-\left(\beta \varrho_{\varepsilon}+\eta_{\varepsilon}\right) \nabla_{x} \Phi
$$

(3.31) $\varepsilon\left[\partial_{t} \eta_{\varepsilon}+\operatorname{div}_{x}\left(\eta_{\varepsilon} \mathbf{u}_{\varepsilon}\right)\right]-\operatorname{div}_{x}\left(\zeta \eta_{\varepsilon} \nabla_{x} \Phi\right)-D \Delta_{x} \eta_{\varepsilon}=0$

(3.32) $\varepsilon \frac{\mathrm{d}}{\mathrm{d} t} \int_{\Omega} \frac{\varepsilon^{2}}{2} \varrho_{\varepsilon}\left|\mathbf{u}_{\varepsilon}\right|^{2}+\frac{a}{\gamma-1} \varrho_{\varepsilon}^{\gamma}+\frac{D \eta_{\varepsilon}}{\zeta} \ln \eta_{\varepsilon}+\left(\beta \varrho_{\varepsilon}+\eta_{\varepsilon}\right) \Phi \mathrm{d} x$

$$
+\varepsilon \int_{\Omega} \varepsilon^{2} \mathbb{S}\left(\nabla_{x} \mathbf{u}_{\varepsilon}\right): \nabla_{x} \mathbf{u}_{\varepsilon} \mathrm{d} x+\int_{\Omega}\left|D \frac{\nabla_{x} \eta_{\varepsilon}}{\sqrt{\zeta \eta_{\varepsilon}}}+\sqrt{\zeta \eta_{\varepsilon}} \nabla_{x} \Phi\right|^{2} \mathrm{~d} x \leq 0
$$

Now, assuming $\left\{\varrho_{\varepsilon}, \mathbf{u}_{\varepsilon}, \eta_{\varepsilon}\right\}$ have the following expansions

$$
\begin{aligned}
& \varrho_{\varepsilon}=\tilde{\varrho}+\sum_{i=1}^{\infty} \varepsilon^{i} \varrho_{\varepsilon}^{(i)} \\
& \eta_{\varepsilon}=\tilde{\eta}+\sum_{i=1}^{\infty} \varepsilon^{i} \eta_{\varepsilon}^{(i)} \\
& \mathbf{u}_{\varepsilon}=\tilde{\mathbf{u}}+\sum_{i=1}^{\infty} \varepsilon^{i} \mathbf{u}_{\varepsilon}^{(i)}
\end{aligned}
$$

substition into (3.29)-(3.32) formally yields the target system

(3.33) $g \tilde{\eta}=-\frac{D}{\zeta} \frac{\mathrm{d} \tilde{\eta}}{\mathrm{d} x_{3}}$ 
(3.34) $\frac{\mathrm{d}}{\mathrm{d} x_{3}}\left[a \tilde{\varrho}^{\gamma}\right]=-\beta g \tilde{\varrho}$

(3.35) $\operatorname{div}_{x}(\tilde{\varrho} \tilde{\mathbf{u}})=0$

$$
\tilde{\varrho} \partial_{t} \tilde{\mathbf{u}}+\operatorname{div}_{x}(\tilde{\varrho} \tilde{\mathbf{u}} \otimes \tilde{\mathbf{u}})+\nabla_{x} \Pi=\mu \Delta_{x} \tilde{\mathbf{u}}+\lambda \nabla_{x} \operatorname{div}_{x} \tilde{\mathbf{u}}-\left(\beta \varrho^{(2)}+\eta^{(2)}\right) \nabla_{x} \Phi .
$$

These formal analysis above suggest the following theorem which is proven rigorously in [4].

Theorem 3.5 (Strong stratification limit). Let $(\Omega, \Phi)$ satisfy the confinement hypothesis and for each $\varepsilon>0,\left\{\varrho_{\varepsilon}, \boldsymbol{u}_{\varepsilon}, \eta_{\varepsilon}\right\}$ solves $(3.29)-(3.32)$ in the sense of the definition of the scaled strong stratification system. Assume the initial data can be expressed as follows:

$$
\varrho_{\varepsilon}(0, \cdot)=\varrho_{\varepsilon, 0}=\tilde{\varrho}+\varepsilon \varrho_{\varepsilon, 0}^{(1)}, \boldsymbol{u}_{\varepsilon}(0, \cdot)=\boldsymbol{u}_{\varepsilon, 0}, \text { and } \eta_{\varepsilon}(0, \cdot)=\eta_{\varepsilon, 0}=\tilde{\eta}+\varepsilon \eta_{\varepsilon, 0}^{(1)} \text {, }
$$

where $\tilde{\varrho}, \tilde{\eta}$ are the densities. Assume also that as $\varepsilon \rightarrow 0$,

$$
\varrho_{\varepsilon, 0}^{(1)} \rightarrow \varrho_{0}^{(1)}, \boldsymbol{u}_{\varepsilon, 0} \rightarrow \overline{\boldsymbol{u}}_{0}, \eta_{\varepsilon, 0}^{(1)} \rightarrow \eta_{0}^{(1)}
$$

weakly-* in $L^{\infty}(\Omega)$ or $L^{\infty}\left(\Omega ; \mathbb{R}^{3}\right)$ as the case may be. Then up to a subsequence and letting $q:=\min \{\gamma, 2\}$,

$$
\begin{gathered}
\varrho_{\varepsilon} \rightarrow \tilde{\varrho} \text { in } C\left([0, T] ; L^{1}(\Omega)\right) \cap L^{\infty}\left(0, T ; L^{q}(\Omega)\right) \\
\eta_{\varepsilon} \rightarrow \tilde{\eta} \text { in } L^{2}\left(0, T ; L^{2}(\Omega)\right) \\
\boldsymbol{u}_{\varepsilon} \rightarrow \tilde{\boldsymbol{u}} \text { weakly in } L^{2}\left(0, T ; W^{1,2}\left(\Omega ; \mathbb{R}^{3}\right)\right)
\end{gathered}
$$

where $\{\tilde{\varrho}, \tilde{\boldsymbol{u}}, \tilde{\eta}\}$ solve the target system (3.33)-(3.36) weakly.

\section{Inviscid models: main results}

\subsection{Reformulation of the problem}

In this section we provide a reformulation of the Cauchy problem for (1.7)(1.9) with the initial condition (1.10). The objective is to transform the original system into a system which includes a symmetric system. In the spirit of [40] we introduce the sound speed

$$
\sigma(\varrho)=\sqrt{p^{\prime}(\varrho)},
$$


and set $\bar{\sigma}=\sigma(\bar{\varrho})$ corresponding to the sound speed at a background density $\bar{\varrho}>0$. Define

$$
w:=\frac{2}{\gamma-1}[\sigma(\varrho)-\bar{\sigma}]
$$

Then the system (1.7)-(1.9) are transformed into the following system for $C^{1}$ solutions:

$$
\begin{gathered}
\partial_{t} w+\bar{\sigma} \operatorname{div}_{x} \mathbf{u}=-\mathbf{u} \cdot \nabla_{x} w-\frac{\gamma-1}{2} w \operatorname{div}_{x} \mathbf{u} \\
\partial_{t} \mathbf{u}+\bar{\sigma} \nabla_{x} w+\beta \nabla_{x} \Phi=-\mathbf{u} \cdot \nabla_{x} \mathbf{u}-\frac{\gamma-1}{2} w \nabla_{x} w \\
-f(w)\left(\nabla_{x} \eta+\eta \nabla_{x} \Phi\right) \\
\partial_{t} \eta+\eta \operatorname{div}_{x} \mathbf{u}-\operatorname{div}_{x}\left(\eta \nabla_{x} \Phi\right)=-\mathbf{u} \cdot \nabla_{x} \eta+\Delta_{x} \eta
\end{gathered}
$$

where $f(w)=\varrho^{-1}$.

After this transformation is done, it is clear that if $\{\varrho, \mathbf{u}, \eta\}$ solve (1.7)(1.9), then the corresponding $\{w, \mathbf{u}, \eta\}$ through the transformation above solve (4.1), and vice versa, which is highlighted in the following lemmas.

Lemma 4.1. For any $T>0$, if $\{\varrho, \boldsymbol{u}\} \in C^{1}\left(\mathbb{R}^{3} \times[0, T]\right)$ is a solution of $(1.7)$ with $\varrho>0$, then $\{w, \boldsymbol{u}\} \in C^{1}\left(\mathbb{R}^{3} \times[0, T]\right)$ is a solution of (4.1)-(4.3) with $\frac{\gamma-1}{2} w+\bar{\sigma}>0$. Conversely, if $\{w, \boldsymbol{u}\} \in C^{1}\left(\mathbb{R}^{3} \times[0, T]\right)$ is a solution of (4.1) with $\frac{\gamma-1}{2} w+\bar{\sigma}>0$ and $\varrho=\sigma^{-1}\left(\frac{\gamma-1}{2} w+\bar{\sigma}\right)$, then $\{\varrho, \boldsymbol{u}\} \in C^{1}\left(\mathbb{R}^{3} \times[0, T]\right)$ is a solution of the continuity equation (1.7) with $\varrho>0$.

The continuity of the density is a consequence of the positivity of the initial density.

Lemma 4.2. If $\{\varrho, \boldsymbol{u}, \eta\} \in C^{1}\left(\mathbb{R}^{3} \times[0, T]\right.$ is a uniformly bounded solution of (1.1) with $\varrho(x, 0)>0$, then $\varrho(x, t)>0$ on $\mathbb{R}^{3} \times[0, T]$. If $\{w, \boldsymbol{u}\} \in C^{1}\left(\mathbb{R}^{3} \times\right.$ $[0, T])$ is a uniformly bounded solution of (4.1) with $\frac{\gamma-1}{2} w(x, 0)+\bar{\sigma}>0$, then $\frac{\gamma-1}{2} w(x, t)+\bar{\sigma}>0$ on $\mathbb{R}^{3} \times[0, T]$.

\subsection{Iterative method and local existence}

Equations (4.1)-(4.2) form a symmetric hyperbolic system in the unknowns $w$ and $\mathbf{u}$. Indeed, if we consider $\eta$ to be given, we can rewrite (4.1)-(4.2) as

$$
\partial_{t} V+\sum_{i=1}^{3} \mathbb{B}_{i} \partial_{x_{i}} V+\mathbb{B}_{4}
$$


where

$$
\begin{aligned}
& V:=\left[\begin{array}{c}
w \\
\mathbf{u}_{1} \\
\mathbf{u}_{2} \\
\mathbf{u}_{3}
\end{array}\right] \\
& \mathbb{B}_{1}:=\left[\begin{array}{cccc}
\mathbf{u}_{1} & \bar{\sigma}+\frac{\gamma-1}{2} w & 0 & 0 \\
\bar{\sigma}+\frac{\gamma-1}{2} w & \mathbf{u}_{1} & 0 & 0 \\
0 & 0 & \mathbf{u}_{1} & 0 \\
0 & 0 & 0 & \mathbf{u}_{1}
\end{array}\right] \\
& \mathbb{B}_{2}:=\left[\begin{array}{cccc}
\mathbf{u}_{2} & 0 & \bar{\sigma}+\frac{\gamma-1}{2} w & 0 \\
0 & \mathbf{u}_{2} & 0 & 0 \\
\bar{\sigma}+\frac{\gamma-1}{2} w & 0 & \mathbf{u}_{2} & 0 \\
0 & 0 & 0 & \mathbf{u}_{2}
\end{array}\right] \\
& \mathbb{B}_{3}:=\left[\begin{array}{cccc}
\mathbf{u}_{3} & 0 & 0 & \bar{\sigma}+\frac{\gamma-1}{2} w \\
0 & \mathbf{u}_{3} & 0 & 0 \\
0 & 0 & \mathbf{u}_{3} & 0 \\
\bar{\sigma}+\frac{\gamma-1}{2} w & 0 & 0 & \mathbf{u}_{3}
\end{array}\right],
\end{aligned}
$$

and

$$
\mathbb{B}_{4}:=\left[\begin{array}{c}
0 \\
(\beta+f(w) \eta) \partial_{x_{1}} \Phi+f(w) \partial_{x_{1}} \eta \\
(\beta+f(w) \eta) \partial_{x_{2}} \Phi+f(w) \partial_{x_{2}} \eta \\
(\beta+f(w) \eta) \partial_{x_{3}} \Phi+f(w) \partial_{x_{3}} \eta
\end{array}\right]
$$

Below a sequence $\left\{w^{k}, \mathbf{u}^{k}, \eta^{k}\right\}$ of approximate solutions to (4.1)-(4.3) is constructed.

An approximate solution $\eta^{1}$ is found by using a solution to a heat equation. Then, $\eta^{1}$ will be substituted into (4.1)-(4.3) to obtain $\mathbf{u}^{1}$ and $w^{1}$. Then, $\mathbf{u}^{1}$ will be substituted into (4.3) to solve for $\eta^{2}$, which will be plugged into (4.1)-(4.3) to obtain $w^{2}$ and $\mathbf{u}^{2}$, and so on, continuing inductively. To begin, consider the Cauchy problem

$$
\begin{aligned}
& \partial_{t} \mathbf{v}-\Delta_{x} \mathbf{v}=0 \\
& \mathbf{v}(x, 0)=\mathbf{u}_{0}
\end{aligned}
$$

In order to be able to use the theorem for local existence of the symmetric hyperbolic system (4.1)-(4.2), assume the following regularity on the initial data $w_{0}$ and $\mathbf{u}_{0}$ :

$$
w_{0} \in W^{3,2}(\Omega)
$$




$$
\mathbf{u}_{0} \in W^{3,2}\left(\Omega ; \mathbb{R}^{3}\right) .
$$

Assume also that the support of $w_{0}$ and $\mathbf{u}_{0}$ is within a compact subset of $\Omega$. By the Sobolev embedding theorems, it is clear that

$$
\begin{aligned}
& w_{0} \in C^{1,1 / 2}(\Omega) \\
& \mathbf{u}_{0} \in C^{1,1 / 2}\left(\Omega ; \mathbb{R}^{3}\right) .
\end{aligned}
$$

Because of this, if $\mathbf{u}^{0}$ solves (4.5), then $\mathbf{u}^{0} \in C^{\infty}\left(\Omega ; \mathbb{R}^{3}\right)$ from basic properties of the Cauchy problem of the heat equation (see, for example, Chapter 2.3 in [23]). If $\Phi \in C^{2}(\Omega)$, thus making the coefficients in (4.3) continuous (using $\mathbf{u}^{0}$ for $\mathbf{u}$ ), the results in Chapter 7.1 of [23] yield a solution

$$
\eta^{1} \in C^{1}\left([0, T] ; C^{2}(\Omega)\right) .
$$

Consider the approximation of the system (4.1)-(4.3):

$$
\begin{aligned}
& \partial_{t} w^{k}+\bar{\sigma} \operatorname{div}_{x} \mathbf{u}^{k}=-\mathbf{u}^{k} \cdot \nabla_{x} w^{k}-\frac{\gamma-1}{2} w^{k} \operatorname{div}_{x} \mathbf{u}^{k} \\
& \partial_{t} \mathbf{u}^{k}+\bar{\sigma} \nabla_{x} w^{k}+\left[\beta+f\left(w^{k}\right) \eta^{k}\right] \nabla_{x} \Phi+f(w) \nabla_{x} \eta \\
& =-\left(\mathbf{u}^{k} \cdot \nabla_{x}\right) \mathbf{u}^{k}-\frac{\gamma-1}{2} w^{k} \nabla_{x} w^{k} \\
& \partial_{t} \eta^{k}+\eta^{k} \operatorname{div}_{x}\left(\mathbf{u}^{k-1}-\nabla_{x} \Phi\right)+\left(\mathbf{u}^{k-1}-\nabla_{x} \Phi\right) \cdot \nabla_{x} \eta^{k}-\Delta \eta^{k}=0 .
\end{aligned}
$$

Next, adapting Theorem 2.1 from [35] used also in [34] and [40] on the existence of smooth solutions for local time of symmetric hyperbolic systems to this problem we get the following theorem.

Theorem 4.3 (Solutions for Symmetric Hyperbolic Systems). Let $w_{0} \in$ $W^{3,2}(\Omega)$ and $\boldsymbol{u}_{0} \in W^{3,2}\left(\Omega ; \mathbb{R}^{3}\right)$ with the support of $w_{0}$ and $\boldsymbol{u}_{0}$ contained in some compact subset $K$ of $\Omega$. Assume also that $\eta^{k} \in C^{1}\left([0, T] ; C^{2}(\Omega)\right)$. Then there is a time interval $[0, T]$ with $T>0$ such that there is a unique classical solution

$$
\begin{aligned}
& w^{k} \in C\left([0, T] ; W^{3,2}(\Omega)\right) \cap C^{1}\left([0, T] ; W^{2,2}(\Omega)\right) \\
& \boldsymbol{u}^{k} \in C\left([0, T] ; W^{3,2}\left(\Omega ; \mathbb{R}^{3}\right)\right) \cap C^{1}\left([0, T] ; W^{2,2}\left(\Omega ; \mathbb{R}^{3}\right)\right) .
\end{aligned}
$$

Furthermore, $T$ depends only on $w_{0}, \boldsymbol{u}_{0}$ and $K$.

Proof. The proof of the local existence of solutions proceeds via a classical iteration scheme in the spirit of Majda [35]. It relies on the elementary 
linear existence theory for symmetric hyperbolic systems with smooth coefficients (Courant-Hilbert [18]. We refer the reader also to the article by Sideris et al. [40] where the local existence result for a related system is presented.

Remark 4.4. Due to the Sobolev embedding theorems,

$$
\begin{aligned}
& w^{k} \in C^{1}\left([0, T] ; C^{0,1 / 2}(\Omega)\right) \cap C\left([0, T] ; C^{1,1 / 2}(\Omega)\right) \\
& \mathbf{u}^{k} \in C^{1}\left([0, T] ; C^{0,1 / 2}\left(\Omega ; \mathbb{R}^{3}\right)\right) \cap C\left([0, T] ; C^{1,1 / 2}\left(\Omega ; \mathbb{R}^{3}\right)\right)
\end{aligned}
$$

Note that Theorem 4.3 implies that the maximal time of existence will be the same positive number $T$ for each $k$ in the sequence. Thus when taking the limit, there is no worry about the limiting maximal time of existence being zero. Now, the regularity of $\mathbf{u}^{k}$, can be used in (4.9) to obtain $\eta^{k+1} \in$ $C^{1}\left([0, T] ; C^{2}(\Omega)\right)$, which is then used with Theorem 4.3 to obtain $w^{k+1}$ and $\mathbf{u}^{k+1}$, giving one of the main theorem of this section:

Theorem 4.5 (Existence of Approximate Smooth Solutions). Let

$$
\begin{aligned}
& w_{0} \in W^{3,2}(\Omega) \\
& \boldsymbol{u}_{0} \in W^{3,2}\left(\Omega ; \mathbb{R}^{3}\right) \\
& \eta_{0} \in W^{3,2}(\Omega)
\end{aligned}
$$

all with support contained in some compact subset $K$ of $\Omega$. Let

$$
\boldsymbol{u}^{0} \in C^{1}\left([0, T] ; C^{2}(\Omega)\right)
$$

solve (4.5). Then there exists some $T>0$ such that for all $k \in \mathbb{N}$, there exist solutions $\left\{w^{k}, \boldsymbol{u}^{k}, \eta^{k}\right\}$ of (4.7)-(4.9) such that

$$
\begin{aligned}
w^{k} & \in C\left([0, T] ; W^{3,2}(\Omega)\right) \cap C^{1}\left([0, T] ; W^{2,2}(\Omega)\right) \\
\boldsymbol{u}^{k} & \in C\left([0, T] ; W^{3,2}\left(\Omega ; \mathbb{R}^{3}\right)\right) \cap C^{1}\left([0, T] ; W^{2,2}\left(\Omega ; \mathbb{R}^{3}\right)\right) \\
\eta^{k} & \in C^{1}\left([0, T] ; C^{2}(\Omega)\right) .
\end{aligned}
$$

Furthermore, $T$ depends only on $w_{0}, \boldsymbol{u}_{0}$ and $K$.

Proof. The result follows from Theorem 4.3, the discussion on the existence of $\mathbf{u}^{0}$ and $\eta^{1}$, and the induction argument outlined above.

Now, we take $k \rightarrow \infty$ to obtain a solution to (4.1)-(4.3). By using an iteration technique, we show that the approximate solutions converge and we obtain the local existence of smooth solutions to (4.1)-(4.3) 
Theorem 4.6 (Local Existence of Smooth Solutions). Let

$$
\begin{aligned}
& w_{0} \in W^{3,2}(\Omega) \\
& \boldsymbol{u}_{0} \in W^{3,2}\left(\Omega ; \mathbb{R}^{3}\right) \\
& \eta_{0} \in W^{3,2}(\Omega)
\end{aligned}
$$

all with support contained in some compact subset $K$ of $\Omega$. Then for some $T>0$, there exist solutions $\{\varrho, \boldsymbol{u}, \eta\}$ of (4.1)-(4.3) such that

$$
\begin{aligned}
& \varrho \in C\left([0, T] ; W^{3,2}(\Omega)\right) \cap C^{1}\left([0, T] ; W^{2,2}(\Omega)\right) \\
& \boldsymbol{u} \in C\left([0, T] ; W^{3,2}\left(\Omega ; \mathbb{R}^{3}\right)\right) \cap C^{1}\left([0, T] ; W^{2,2}\left(\Omega ; \mathbb{R}^{3}\right)\right) \\
& \eta \in C^{1}\left([0, T] ; C^{2}(\Omega)\right) .
\end{aligned}
$$

\section{Finite propagation speed}

Consider the nonlinear balance law

$$
\partial_{t} U+\operatorname{div}_{x}(F(U))=G(U)
$$

where $U=U(x, t) \in \mathbb{R}^{n}$ and $G(U): \mathbb{R}^{n} \rightarrow \mathbb{R}^{n}$. We remark, that the inviscid system (1.7)-(1.8) can be written in this form, with

$$
U=(\varrho, \mathbf{u})^{\top} \in \mathbb{R}^{4}, \quad G(U)=\left(0,-(\eta+\beta \varrho) \nabla_{x} \Phi\right)^{\top} \in \mathbb{R}^{4} .
$$

Here, and in what follows, we require that the Euler system (1.7)-(1.8) is weakly dissipative in the sense of Dafermos [22], which requires that

$$
(G(U), U) \leq 0, \quad U \in \mathbb{R}^{4}
$$

In the case, of the inviscid system (1.7)-(1.8),

$$
(G(U), U)=\left(0,-(\eta+\beta \varrho) \nabla_{x} \Phi \cdot \mathbf{u}\right)^{\top}
$$

The assumption ( $\mathrm{w}-\mathrm{d}$ ) essentially imposes an additional requirement on the class of potentials $\Phi$, which are now chosen so that to induce weak dissipation for the Euler system (1.7)-(1.8) in the sense of Dafermos [22], namely

$$
-(\eta+\beta \varrho) \nabla_{x} \Phi \cdot \mathbf{u} \leq 0
$$


This assumption on the potential $\Phi$ will be of use in the sequel and in fact crucial for the establishment of the global existence of smooth solutions.

Let us denote now,

$$
U(x, t)=(w(x, t), \mathbf{u}(x, t), \eta(x, t))
$$

with $U_{0}(x)=U(x, 0)$ the initial data. We will say that $U(\cdot, t) \in H^{3}$ if $w(\cdot, t) \in W^{3,2}\left(\mathbb{R}^{3}\right), \mathbf{u}(\cdot, t) \in W^{3,2}\left(\mathbb{R}^{3} ; \mathbb{R}^{3}\right)$, and $\eta(\cdot, t) \in C^{2}\left(\mathbb{R}^{3}\right)$. Similarly, we will say that $U(\cdot, t) \in H^{2}$ if $w(\cdot, t) \in W^{2,2}\left(\mathbb{R}^{3}\right), \mathbf{u}(\cdot, t) \in W^{2,2}\left(\mathbb{R}^{3} ; \mathbb{R}^{3}\right)$, and $\eta(\cdot, t) \in C^{2}\left(\mathbb{R}^{3}\right)$.

The property of the finite speed of propagation of the solution now follows.

Lemma 5.1. Suppose that $U_{0} \in H^{3}$ and $U \in C\left([0, T], H^{3}\right) \cap C^{1}\left([0, T], H^{2}\right)$ is a solution to the Cauchy problem (4.1)- (4.3) for any given $T>0$. If $\operatorname{supp} U_{0} \subset\{|x| \leq R\}$, for some $R>0$, then $\operatorname{supp} U(\cdot, t) \subset\{|x| \leq R+\bar{\sigma} t\}$, for $0 \leq t<T$.

Proof. Multiplying (4.1) by $w,(4.2)$ by $u$ and (4.3) by $\eta$ and adding the resulting relations together we get

$$
\begin{aligned}
& \frac{1}{2} \partial_{t} w^{2}+\frac{1}{2} \partial_{t}|\mathbf{u}|^{2}+\frac{1}{2} \partial_{t} \eta^{2}+\bar{\sigma} \operatorname{div}_{x}(w \mathbf{u}) \\
& +\frac{1}{2}\left(\mathbf{u} \cdot \nabla\left(|\mathbf{u}|^{2}+w^{2}+\eta^{2}\right)+(\gamma-1) w \operatorname{div}_{x}(w \mathbf{u})\right)+\eta^{2} \operatorname{div}_{x} \mathbf{u} \\
& =\eta \nabla_{x} \Phi \cdot \nabla_{x} \eta-\beta \mathbf{u} \cdot \nabla_{x} \Phi-\eta f(w) \mathbf{u} \cdot \nabla_{x} \Phi-f(w) \mathbf{u} \cdot \nabla_{x} \eta+\eta \Delta_{x} \eta
\end{aligned}
$$

For a given $(x, t) \in \mathbb{R}^{3} \times(0, T]$, take any $\tau \in[0, t)$, and define the truncated cone

$$
C_{\tau}=\{(y, s):|y-x| \leq \bar{\sigma}(t-s), 0 \leq s \leq \tau\} .
$$

Integrating (5.3) over $C_{\tau}$ we arrive at

$$
\begin{aligned}
& \frac{1}{2} \int_{|y-x| \leq \bar{\sigma}(t-\tau)}\left(w^{2}+|\mathbf{u}|^{2}+\eta^{2}\right)(y, \tau) \mathrm{d} y-\frac{1}{2} \int_{|y-x| \leq \bar{\sigma} t}\left(w^{2}+|\mathbf{u}|^{2}+\eta^{2}\right)(y, 0) \mathrm{d} y \\
& +\frac{1}{\sqrt{\bar{\sigma}^{2}+1}} \int_{0}^{\tau} \int_{|y-x|=\bar{\sigma}(t-\tau)}\left(\frac{\bar{\sigma}}{2}\left(w^{2}+|\mathbf{u}|^{2}+\eta^{2}\right)+\frac{y-x}{|y-x|} \cdot \bar{\sigma} w \mathbf{u}\right) \mathrm{d} S_{y} \mathrm{~d} s \\
& \quad-\frac{\bar{\sigma}}{\sqrt{\bar{\sigma}^{2}+1}} \int_{0}^{\tau} \int_{|y-x|=\bar{\sigma}(t-s)}\left(\eta^{2} \nabla_{x} \Phi+\eta \nabla_{x} \eta\right) \cdot \frac{y-x}{|y-x|} \mathrm{d} S_{y} \mathrm{~d} s \\
& =\iint_{C_{\tau}}\left(-\frac{1}{2}\left(\mathbf{u} \cdot \nabla\left(w^{2}+|\mathbf{u}|^{2}\right)+(\gamma-1) w \operatorname{div}_{x}(w \mathbf{u})\right)\right) \mathrm{d} y \mathrm{~d} s
\end{aligned}
$$


$+\iint_{C_{\tau}} \eta \nabla_{x} \Phi \cdot \nabla_{x} \eta-\beta \mathbf{u} \cdot \nabla_{x} \Phi-\eta f(w) \mathbf{u} \cdot \nabla_{x} \Phi-f(w) \mathbf{u} \cdot \nabla_{x} \eta-\left|\nabla_{x} \eta\right|^{2} \mathrm{~d} y \mathrm{~d} s$

The third integral in the left hand side of (5.4) is nonnegative in account of

$$
\left|\frac{y-x}{|y-x|} \cdot \bar{\sigma} w \mathbf{u}\right| \leq \bar{\sigma}\left(w^{2}+|\mathbf{u}|^{2}\right) .
$$

Using integration by parts, the inequality $|w \mathbf{u}| \leq \frac{1}{2}\left(w^{2}+|\mathbf{u}|^{2}\right)$ and CauchySchwarz inequality we get

$$
\begin{aligned}
& \frac{1}{2} \int_{|y-x| \leq \bar{\sigma}(t-\tau)} w^{2}+|\mathbf{u}|^{2}+\eta^{2} \mathrm{~d} y-\frac{1}{2} \int_{|y-x| \leq \bar{\sigma} t} w^{2}+|\mathbf{u}|^{2}+\eta^{2} \mathrm{~d} y \\
& +\bar{\sigma} \int_{0}^{\tau} \int_{|y-x|=\bar{\sigma}(t-s)} \frac{1}{2}\left(w^{2}+|\mathbf{u}|^{2}+\eta^{2}\right)+w \mathbf{u} \cdot \frac{y-x}{|y-x|} \mathrm{d} S_{y} \mathrm{~d} s \\
& -\frac{\bar{\sigma}}{\sqrt{\bar{\sigma}^{2}+1}} \int_{0}^{\tau} \int_{|y-x|=\bar{\sigma}(t-s)}\left(\eta^{2} \nabla_{x} \Phi+\eta \nabla_{x} \eta\right) \cdot \frac{y-x}{|y-x|} \mathrm{d} S_{y} \mathrm{~d} s \\
& \leq \int_{0}^{\tau} \int_{|y-x| \leq \bar{\sigma}(t-s)} \frac{1}{2} \eta \nabla_{x} \Phi \cdot \nabla_{x} \eta-\eta^{2} \operatorname{div}_{x} \mathbf{u}-\beta \mathbf{u} \cdot \nabla_{x} \Phi \mathrm{d} y \mathrm{~d} s \\
& -\int_{0}^{\tau} \int_{|y-x| \leq \bar{\sigma}(t-s)} \eta f(w) \mathbf{u} \cdot \nabla_{x} \Phi+f(w) \mathbf{u} \cdot \nabla_{x} \eta+f(w) \eta \mathbf{u} \cdot \nabla_{x} \Phi \mathrm{d} y \mathrm{~d} s \\
& -\int_{0}^{\tau} \int_{|y-x| \leq \bar{\sigma}(t-s)} \frac{\gamma-1}{2} w \mathbf{u} \cdot \nabla_{x} w+\frac{\gamma-1}{2} w^{2} \operatorname{div}_{x} \mathbf{u} \mathrm{d} y \mathrm{~d} s \\
& -\int_{0}^{\tau} \int_{|y-x| \leq \bar{\sigma}(t-s)}^{\mathbf{u} \cdot\left(\mathbf{u} \cdot \nabla_{x} \mathbf{u}\right)+\eta \mathbf{u} \cdot \nabla_{x} \eta \mathrm{d} y \mathrm{~d} s .}
\end{aligned}
$$

Thus, if the background density $\varrho$ is chosen such that $2\left\|\nabla_{x} \Phi\right\|_{L^{\infty}\left(\Omega ; \mathbb{R}^{3}\right)} \leq$ $\bar{\sigma}$, if $\varrho^{-1}$ is bounded in $L^{1}\left(0, T ; L^{\infty}(\Omega)\right)$ and the weak dissipation relation $-(\beta \varrho+\eta) \nabla_{x} \Phi \cdot \mathbf{u} \leq 0$ holds, Gronwall's Lemma allows us to conclude the finite propagation of the transformed system.

Therefore, if $U(z, 0)=0$ for $|z-x| \leq \bar{\sigma} t$, then $U(z, \tau)=0$ for $|z-x| \leq$ $\bar{\sigma}(t-\tau)$ and any $\tau \in[0, t)$. This implies that if $U(x, 0)=0$ for $|x|>R$ then $U(x, t)=0$ for $|x|>R+\bar{\sigma} t$.

The global existence of smooth solutions is now obtained after deriving appropriate energy estimates. The details of the analysis as well as results on the decay of solutions are presented in [7]. 


\section{Acknowlegments}

The work of J.B. was supported by the National Science Foundation under the grant DMS-1211638 and the Ann G. Wylie Dissertation Fellowship. Trivisa gratefully acknowledges the support in part by the National Science Foundation under the grant DMS-1211519 and by the Simons Foundation under the Simons Fellows in Mathematics Award 267399.

\section{References}

[1] A. A. Amsden. Kiva-3V Release 2, Improvements to Kiva-3V. Tech. Rep., Los Alamos National Laboratory (1999).

[2] A. A. Amsden, P. J. O'Rourke and T. D. Butler. Kiva-2, a computer program for chemical reactive flows with sprays. Tech. Rep., Los Alamos National Laboratory (1989).

[3] J. Ballew. Local in time existence of smooth solutions to the NavierStokes-Smoluchowski system for compressible fluids. Preprint, 2013.

[4] J. Ballew. Low Mach number limits to the Navier-Stokes-Smoluchowski system. Hyperbolic Problems: Theory, Numerics, Applications. AIMS Series on Applied Mathematics, Vol. 8, 2014, pp. 301-308.

[5] J. Ballew and K. Trivisa. Suitable weak solutions and low stratification singular limit for a fluid particle interaction model. Quart. Appl. Math. 70 (2012), 469-494. MR2986131

[6] J. Ballew and K. Trivisa. Weakly dissipative solutions and weakstrong uniqueness for the Navier-Stokes-Smoluchowski system. Nonlinear Analysis Series A: Theory, Methods $\&$ Applications 91 (2013), 1-19. MR3081207

[7] J. Ballew and K. Trivisa. On the dynamics of an Euler fluid particle interaction system: Existence and decay of solutions. Preprint (2013).

[8] C. Baranger. Modélisation, étude mathématique et simulation des collisions dans les fluides complexes. Théses ENS Cachan, Juin 2004.

[9] C. Baranger, L. Boudin, P.-E. Jabin, and S. Mancini. A modeling of biospray for the upper airways. CEMRACS 2004-mathematics and applications to biology and medicine, ESAIM Proc. 14 (2005), 41-4. MR2226800

[10] S. Berres, R. Bürger, K. H. Karlsen, and E. M. Rory. Strongly degenerate parabolic-hyperbolic systems modeling polydisperse sedimentation with compression. SIAM J. Appl. Math. 64 (2003), 41-80. MR2029124 
[11] F. Berthelin and A. Vasseur. From Kinetic Equations to Multidimensional Isentropic Gas Dynamics Before Shocks, SIAM J. Math. Anal. 36 (2005), 1807-1835. MR2178222

[12] L. Boudin, L. Desvillettes, and R. Motte. A modeling of compressible droplets in a fluid. Commun. Math. Sci. 1 (2003), 657-669. MR2041452

[13] J. A. Carrillo and T. Goudon. Stability and Asymptotic Analysis of a Fluid-Particle Interaction Model. Comm. Partial Differential Equations 31 (2006), 1349-1379. MR2254618

[14] J. A. Carrillo, T. Goudon, and P. Lafitte. Simulation of fluid and particles flows: asymptotic preserving schemes for bubbling and flowing regimes. J. Comput. Phys. 227 (2008), 7929-7951. MR2437595

[15] J. A. Carrillo, T. Karper, and K. Trivisa. On the dynamics of a fluidparticle interaction model: The bubbling regime. Nonlinear Analysis $\mathbf{7 4}$ (2011), 2778-2801. MR2776527

[16] G.-Q. Chen and D. Wang. The Cauchy problem for the Euler equations for compressible fluids. Handbook of Mathematical Fluid Dynamics I (2002), 421-543. MR1942468

[17] R. Courant and K. O. Friedrichs. Supersonic Flow and Shock Waves, Springer-Verlag, New York, 1962. MR0421279

[18] R. Courant and D. Hilbert. Methods of Mathematical Physics, Interscience Publishers, Inc., New York, 1953. MR0065391

[19] C. Dafermos. The second law of thermodynamics and stability. Arch. Rational Mech. Anal. 70 (1979), 167-179. MR0546634

[20] C. M. Dafermos. Hyperbolic Conservation Laws in Continuum Physics, 3rd ed. Springer, Berlin, 2010. MR2574377

[21] R. DiPerna. Uniqueness of Solutions to Hyperbolic Conservation Laws, Indiana Univ. Math. J. 28(1) (1979), 137-188. MR0523630

[22] C. M. Dafermos. Hyperbolic systems of balance laws with weak dissipation, Journal of Hyperbolic Differential Equations 3(3) (2006), 505-527. MR2238740

[23] L. C. Evans. Partial differential equations. Graduate Studies in Mathematics, No. 19. American Mathematical Society, Providence, 1998. MR1625845

[24] J. Dolbeault. Free energy and solutions of the Vlasov-Poisson-FokkerPlanck system: external potential and confinement (large time be- 
havior and steady states), J. Math. Pures Appl. 78 (1999), 121-157. MR1677677

[25] E. Feireisl. Dynamics of viscous compressible fluids. Oxford University Press, Oxford, 2003. MR2040667

[26] E. Feireisl and A. Novotný. Singular Limits in Thermodynamics of Viscous Fluids. Birkhäuser, Basel, 2009. MR2499296

[27] E. Feireisl, A. Novotný, and H. Petzeltová. On the existence of globally defined weak solutions to the Navier-Stokes equations of compressible isentropic fluids. J. Math. Fluid Dynamics 3 (2001), 358-392. MR1867887

[28] E. Feireisl, B. J. Jin, and A. Novotný. Relative entropies, suitable weak solutions, and weak-strong uniqueness for the compressible NavierStokes system. J. Math. Fluid Mech. 14 (2012), 717-730. MR2992037

[29] E. Feireisl, A. Novotný, and Y. Sun. Suitable weak solutions to the Navier-Stokes equations of compressible viscous fluids. Nečas Center for Mathematical Modeling, Preprint 2010-019, 2010. MR2963786

[30] P. Germain. Weak-strong uniqueness for the isentropic Compressible Navier-Stokes system. J. Math. Fluid Mech. 13 (2011), 137-146. MR2784900

[31] Th. Goudon, P.-E. Jabin, and A. Vasseur. Hydrodynamic limit for the Vlasov-Navier-Stokes equations. I. Light particles regime. Indiana Univ. Math. J. 53(6) (2004), 1495-1515. MR2106333

[32] Th. Goudon, P.-E. Jabin, and A. Vasseur. Hydrodynamic limit for the Vlasov-Navier-Stokes equations. II. Fine particles regime. Indiana Univ. Math. J. 53(6) (2004), 1517-1536. MR2106334

[33] T. Kato. Wave operators and similarity for some non-selfadjoint operators. Math. Ann. 162 (1965/1966), 258-279. MR0190801

[34] T. Kato. The Cauchy problem for quasi-linear symmetric hyperbolic systems. Arch. Rational Mech. Anal. 58 (1975), 181-205. MR0390516

[35] A. Majda. Compressible fluid flow and systems of conservation laws in several space variables, Applied Mathematical Sciences 53, SpringerVerlag, New York, 1984. MR0748308

[36] A. Mellet and A. Vasseur. Asymptotic analysis for a Vlasov-FokkerPlanck/compressible Navier-Stokes system of equations. Comm. Math. Phys. 281 (2008), 573-596. MR2415460 
[37] T. C. Sideris. Formation of singularities in three-dimensional compressible fluids, Commun. Math. Phys. 101 (1985), 475-485. MR0815196

[38] T. C. Sideris. The lifespan of smooth solutions to the three-dimensional compressible Euler equations and the incompressible limit, Indiana Univ. Math. J. 40 (1991), 535-550. MR1119187

[39] T. C. Sideris. Delayed singularity formation in 2d compressible flow, Am. J. Math. 119(2) (1997), 371-422. MR1439554

[40] T. C. Sideris, B. Thonases and D. Wamg. Long time behavior of solutions to the 3D compressible Euler equations with damping. Comm. Partial Diff. Eqs. 28(3,4) (2003), 795-816. MR1978315

[41] I. Vinkovic, C. Aguirre, S. Simoens, and M. Gorokhovski. Large eddy simulation of droplet dispersion for inhomogeneous turbulent wall flow. International Journal of Multiphase Flow 32 (2006), 344-364.

[42] F. A. Williams. Combustion theory, 2nd ed. Benjamin Cummings Publ., 1985.

[43] F. A. Williams. Spray combustion and atomization. Physics of Fluids 1 (1958), 541-555.

JOSHUA BALLEW

DEPARTMENT OF MATHEMATICS

UNIVERSITY OF MARYLAND

College Park, MD 20742

USA

E-mail address: jballew@math.umd.edu

URL: http://www.math.umd.edu/ jballew

Konstantina Trivisa

Department of Mathematics

UNIVERSITY OF MARYLAND

College Park, MD 20742

USA

E-mail address: trivisa@math.umd.edu

URL: http://www. math.umd.edu/ ${ }^{\text {trivisa }}$

Received September 3, 2013 